\title{
Size effect on phonon hydrodynamics in graphite microstructures and nanostructures
}

\author{
Yangyu Guo $\odot,{ }^{1, *}$ Zhongwei Zhang, ${ }^{1}$ Marc Bescond,${ }^{2}$ Shiyun Xiong, ${ }^{1}$ Moran Wang, ${ }^{3}$ \\ Masahiro Nomura, ${ }^{1, \dagger}$ and Sebastian Volz ${ }^{1,2, 末}$ \\ ${ }^{1}$ Institute of Industrial Science, The University of Tokyo, Tokyo 153-8505, Japan \\ ${ }^{2}$ LIMMS, CNRS-IIS UMI 2820, The University of Tokyo, Tokyo 153-8505, Japan \\ ${ }^{3}$ Department of Engineering Mechanics, Tsinghua University, Beijing 100084, China
}

(Received 7 June 2021; revised 20 July 2021; accepted 17 August 2021; published 30 August 2021)

\begin{abstract}
The understanding of hydrodynamic heat transport in finite-sized graphitic materials remains elusive due to the lack of an efficient methodology. In this paper, we develop a computational framework enabling an accurate description of heat transport in anisotropic graphite ribbons by a kinetic theory approach with full quantum mechanical first-principles input. A unified analysis of the size scaling of the thermal conductivity in the longitudinal and transverse directions of the system is made within the computational framework complemented with a macroscopic hydrodynamic approach. As a result, we demonstrate a strong end effect on the phonon Knudsen minimum, as a hallmark of the transition from ballistic to hydrodynamic heat transports, along a rectangular graphite ribbon with finite length and width. The phonon Knudsen minimum is found to take place only when the ribbon length is $\sim 5-10$ times the upper limit of the width range in the hydrodynamic regime. This paper contributes to a unique methodology with high efficiency and a deeper understanding of the size effect on phonon hydrodynamics, which would open opportunities for its theoretical and experimental investigation in graphitic micro- and nanostructures.
\end{abstract}

DOI: 10.1103/PhysRevB.104.075450

\section{INTRODUCTION}

Hydrodynamic heat transport is a collective phenomenon in condensed matter in the presence of dominant momentumconserving normal scattering of phonons [1-3]. The investigation of phonon hydrodynamics was motivated by the exploration of second sound (wavelike heat transport) in solids at low temperatures $[4,5]$. On the other hand, it has significantly promoted the development of macroscopic hydrodynamic equations for non-Fourier heat transport [5-9]. There is a renewed interest in hydrodynamic heat transport in graphitic materials in recent years due to its occurrence at relatively high temperatures [10-12], which shows great potential for thermal management applications [13-15].

The theoretical prediction of phonon hydrodynamics in graphitic materials is mainly based on the homogenous solution of the Boltzmann equation in the bulk limit $[10,11,16]$. To have a deeper understanding of hydrodynamic heat transport in micro- and nanostructures, a direct solution of the space- and time-dependent phonon Boltzmann equation becomes indispensable yet a challenging task. The widely used phonon Boltzmann equation under single mode relaxation time (SMRT) approximation does not work well in this situation due to the collective effect from strong normal scattering $[10,16,17]$. Crucial progress toward this challenge is the Monte Carlo solution of the phonon Boltzmann equation

\footnotetext{
*yyguo@iis.u-tokyo.ac.jp

${ }^{\dagger}$ nomura@iis.u-tokyo.ac.jp

‡volz@iis.u-tokyo.ac.jp
}

with a full scattering term on two-dimensional (2D) reciprocal space [18], which has been mostly applied to study phonon hydrodynamics in 2D graphene ribbons $[3,19]$ due to the model applicability and a considerable computational cost. As the theoretical prediction has been confirmed by the recent experimental reports of second sound [12] and phonon Poiseuille flow [20] in graphite, an efficient methodology for modeling the hydrodynamic heat transport in micro- and nanoribbons of graphite with three-dimensional (3D) reciprocal space is highly desired, which is the main aim of this paper.

Callaway's dual relaxation model [21] represents a good approximation to the full scattering term in the phonon Boltzmann equation $[10,22]$ and has been widely adopted in analyzing heat transport in the hydrodynamic regime by analytical or semi-analytical methods [5,23-29]. The direct solution of the phonon Boltzmann equation under Callaway's model has been advanced recently by a few numerical schemes including both deterministic methods [30,31] and stochastic ones [32,33]. However, the deterministic numerical method [30,31] was designed for heat transport in 2D graphene ribbons with empirical isotropic phonon properties. The gray Monte Carlo simulation [32,33] was conducted in hypothetical graphitic materials due to the lack of knowledge of normal and Umklapp scattering rates and the pending development of the methodology. Thus, none of the previous methods [30-33] are available to describe heat transport in realistic anisotropic graphite ribbons. In this paper, a pertinent computational framework is developed based on a deterministic numerical solution of Callaway's model. The normal and Umklapp phonon scattering rates in graphite are calculated 
through careful consideration of both the van der Waals (vdW) interaction [34] and the special definition of the Umklapp process in strongly anisotropic systems [26,35]. We also introduce an efficient approximate scheme to treat the anisotropic phonon properties of graphite, which is essentially different from the 2D graphene system [30,31]. As a result, our computational framework provides a unique and efficient platform capable of modeling phonon hydrodynamics in graphite micro- and nanostructures.

Within the computational framework, we investigate the hydrodynamic heat transport in graphite ribbon with finite length and width. The in-plane $[19,26,30,36]$ (or crossplane $[28,32]$ ) heat transports along infinitely long (or wide) graphitic ribbons were widely studied previously [3]. A very important hydrodynamic phenomenon, the phonon Knudsen minimum, has been predicted in infinitely long graphene ribbons [30] and graphite ribbons [26]. The phonon Knudsen minimum represents a transition of heat transport from the ballistic regime to the hydrodynamic one, which is definite evidence for the experimental detection of phonon hydrodynamics. However, in the realistic experiment, the graphite ribbon will always have a finite length, the influence of which remains unknown. As another aim of this paper, we will uncover a very strong end effect from the finite length on the phonon Kundsen minimum. The end effect is indeed relevant to the size dependence of the cross-plane thermal conductivity. In comparison with the well-established super-ballistic scaling with the width of in-plane thermal conductivity $[19,26,37,38]$, the size scaling in the cross-plane case remains less clear despite a few notable efforts [28,32]. We will quantify the size scaling of both in-plane and crossplane thermal conductivities in the same footprint based on the direct numerical solution and a hydrodynamic approach, which helps to understand the end effect on phonon Knudsen minimum. The remainder of this article is arranged as follows: The methodology will be introduced in Sec. II, and the results and discussions will be given in Sec. III, with the concluding remarks finally made in Sec. IV.

\section{METHODS}

In Sec. II A, the phonon Boltzmann equation under Callaway's dual relaxation model is firstly introduced. The $a b$ initio phonon properties (dispersion and scattering rates) of graphite are computed and verified in Sec. II B. In Sec. II C, a discrete-ordinate method (DOM) is presented for the numerical solution of the phonon Boltzmann equation under Callaway's model. Finally, a validation of the methodology is demonstrated by comparison with the available semianalytical solution in Sec. II D.

\section{A. Phonon Boltzmann equation under Callaway's model}

The phonon Boltzmann equation under Callaway's scattering model is written as [21,30,31]

$$
\frac{\partial f}{\partial t}+\mathbf{v}_{g} \cdot \nabla f=\frac{f_{\mathrm{R}}^{\mathrm{eq}}\left(T_{\mathrm{loc}, \mathrm{R}}\right)-f}{\tau_{\mathrm{R}}(\mathbf{q}, p, T)}+\frac{f_{\mathrm{N}}^{\mathrm{eq}}\left(T_{\mathrm{loc}, \mathrm{N}}, \mathbf{u}\right)-f}{\tau_{\mathrm{N}}(\mathbf{q}, p, T)},
$$

where $f \equiv f(\mathbf{r}, t, \mathbf{q}, p)$ is the number distribution function of phonon mode (q, $p$ ), with $\mathbf{q}$ and $p$ the wave vector and polar- ization of phonons, respectively. The phonon group velocity is denoted by $\mathbf{v}_{g}$. The equilibrium distribution functions for the intrinsic resistive and normal scattering processes are the Bose-Einstein and the displaced Bose-Einstein distributions separately:

$$
\begin{aligned}
f_{\mathrm{R}}^{\mathrm{eq}} & =\frac{1}{\exp \left(\frac{\hbar \omega}{k_{\mathrm{B}} T_{\mathrm{loc}, \mathrm{R}}}\right)-1}, \\
f_{\mathrm{N}}^{\mathrm{eq}} & =\frac{1}{\exp \left(\frac{\hbar \omega-\hbar \mathbf{q} \cdot \mathbf{u}}{k_{\mathrm{B}} T_{\mathrm{Ioc}, \mathrm{N}}}\right)-1},
\end{aligned}
$$

where $2 \pi \hbar$ and $k_{\mathrm{B}}$ are the Planck and Boltzmann constants, respectively. The two local pseudotemperatures $\left(T_{\mathrm{loc}, \mathrm{R}}, T_{\mathrm{loc}, \mathrm{N}}\right)$ in Eqs. (2) and (3) are intermediate quantities to ensure the local energy conservation conditions of both resistive and normal processes:

$$
\begin{gathered}
\sum_{p} \int \hbar \omega \frac{f_{\mathrm{R}}^{\mathrm{eq}}\left(T_{\mathrm{loc}, \mathrm{R}}\right)-f}{\tau_{\mathrm{R}}(\mathbf{q}, p, T)} \frac{d \mathbf{q}}{(2 \pi)^{3}}=0, \\
\sum_{p} \int \hbar \omega \frac{f_{\mathrm{N}}^{\mathrm{eq}}\left(T_{\mathrm{loc}, \mathrm{N}}, \mathbf{u}\right)-f}{\tau_{\mathrm{N}}(\mathbf{q}, p, T)} \frac{d \mathbf{q}}{(2 \pi)^{3}}=0,
\end{gathered}
$$

and the local phonon drift velocity $\mathbf{u}$ is determined by the local quasimomentum conservation condition of normal process:

$$
\sum_{p} \int \hbar \mathbf{q} \frac{f_{\mathrm{N}}^{\mathrm{eq}}\left(T_{\mathrm{loc}, \mathrm{N}}, \mathbf{u}\right)-f}{\tau_{\mathrm{N}}(\mathbf{q}, p, T)} \frac{d \mathbf{q}}{(2 \pi)^{3}}=0 .
$$

The relaxation times (the inverse of scattering rates) of resistive and normal processes $\left(\tau_{\mathrm{R}}\right.$ and $\left.\tau_{\mathrm{N}}\right)$ are dependent on the local thermodynamic temperature $T$ and will be obtained by the ab initio calculation in the following Sec. II B.

For the convenience of numerical treatment, the deviational energy distribution function of phonon modes is introduced as [39]: $\phi \equiv e-e_{\mathrm{R}}^{\mathrm{eq}}\left(T_{0}\right)=\hbar \omega f-\hbar \omega f_{\mathrm{R}}^{\mathrm{eq}}\left(T_{0}\right)$, with $T_{0}$ the average system temperature. Furthermore, we assume a small temperature difference throughout the system such that the phonon Boltzmann Eq. (1) is linearized as

$$
\frac{\partial \phi}{\partial t}+\mathbf{v}_{g} \cdot \nabla \phi=\frac{\phi_{\mathrm{R}}^{\mathrm{eq}}\left(T_{\mathrm{loc}, \mathrm{R}}\right)-\phi}{\tau_{\mathrm{R}}\left(\mathbf{q}, p, T_{0}\right)}+\frac{\phi_{\mathrm{N}}^{\mathrm{eq}}\left(T_{\mathrm{loc}, \mathrm{N}}, \mathbf{u}\right)-\phi}{\tau_{\mathrm{N}}\left(\mathbf{q}, p, T_{0}\right)},
$$

and the equilibrium distribution functions in Eqs. (2) and (3) become, respectively,

$$
\begin{gathered}
\phi_{\mathrm{R}}^{\mathrm{eq}}\left(T_{\mathrm{loc}, \mathrm{R}}\right)=C_{\mathbf{q} p}\left(T_{\mathrm{loc}, \mathrm{R}}-T_{0}\right), \\
\phi_{\mathrm{N}}^{\mathrm{eq}}\left(T_{\mathrm{loc}, \mathrm{N}}, \mathbf{u}\right)=C_{\mathbf{q} p}\left(T_{\mathrm{loc}, \mathrm{N}}-T_{0}\right)+T_{\mathrm{loc}, \mathrm{N}} C_{\mathbf{q} p} \frac{\mathbf{q} \cdot \mathbf{u}}{\omega},
\end{gathered}
$$

where the modal heat capacity is defined as $C_{\mathbf{q} p}=\left.\hbar \omega \frac{\partial f_{\mathrm{R}}^{\mathrm{eq}}}{\partial T}\right|_{T_{0}}$, whereas the local energy and quasimomentum conservation conditions in Eqs. (4) and (5) are reduced, respectively, to

$$
\begin{array}{r}
T_{\mathrm{loc}, \mathrm{R}}-T_{0}=\frac{1}{C_{\tau_{\mathrm{R}}}} \sum_{p} \int \frac{\phi}{\tau_{\mathrm{R}}\left(\mathbf{q}, p, T_{0}\right)} \frac{d \mathbf{q}}{(2 \pi)^{3}}, \\
T_{\mathrm{loc}, \mathrm{N}}-T_{0}=\frac{1}{C_{\tau_{\mathrm{N}}}} \sum_{p} \int \frac{\phi}{\tau_{\mathrm{N}}\left(\mathbf{q}, p, T_{0}\right)} \frac{d \mathbf{q}}{(2 \pi)^{3}}, \\
u_{\alpha}=\frac{1}{T_{\mathrm{loc}, \mathrm{N}}\left(\mathbf{C}_{\tau_{\mathrm{N}}}^{1}\right)_{\alpha \alpha}} \sum_{p} \int \frac{q_{\alpha}}{\omega} \frac{\phi}{\tau_{\mathrm{N}}\left(\mathbf{q}, p, T_{0}\right)} \frac{d \mathbf{q}}{(2 \pi)^{3}} .
\end{array}
$$


In Eqs. (9) and (10), we have introduced the short notations as $C_{\tau_{\mathrm{R}}}=\sum_{p} \int \frac{C_{\mathbf{q} p}}{\tau_{\mathrm{R}}\left(\mathbf{q}, p, T_{0}\right)} \frac{d \mathbf{q}}{(2 \pi)^{3}}, C_{\tau_{\mathrm{N}}}=\sum_{p} \int \frac{C_{\mathbf{q} p}}{\tau_{\mathrm{N}}\left(\mathbf{q}, p, T_{0}\right)} \frac{d \mathbf{q}}{(2 \pi)^{3}}$, and $\mathbf{C}_{\tau_{\mathrm{N}}}^{1}=\sum_{p} \int \frac{C_{\mathbf{q} p}}{\omega^{2}} \frac{\mathbf{q q}}{\tau_{\mathrm{N}}\left(\mathbf{q}, p, T_{0}\right)} \frac{d \mathbf{q}}{(2 \pi)^{3}}$. The fact that $\mathbf{C}_{\tau_{\mathrm{N}}}^{1}$ is diagonal has been used in deriving Eq. (10), where the subscript $\alpha$ denotes the index of cartesian coordinates $(x, y, z)$. Note that the phonon intensity form of Boltzmann Eq. (1) in our previous work [30] is not adopted here due to the anisotropic phonon properties of graphite.

Once the deviational energy distribution function of phonons in Eq. (6) is resolved, the macroscopic field variables (thermodynamic temperature and heat flux) are computed by a statistical process:

$$
\begin{gathered}
C_{V}\left[T(\mathbf{r}, t)-T_{0}\right]=\sum_{p} \int \phi \frac{d \mathbf{q}}{(2 \pi)^{3}}, \\
\mathbf{J}(\mathbf{r}, t)=\sum_{p} \int \mathbf{v}_{g} \phi \frac{d \mathbf{q}}{(2 \pi)^{3}} .
\end{gathered}
$$

In Eq. (11), the volumetric heat capacity is calculated as

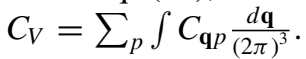

\section{B. Ab initio phonon properties of graphite}

In this subsection, the first-principles calculation of the force constants of graphite are firstly introduced in Sec. II B 1. Then the normal and Umklapp scattering rates are obtained in Sec. II B 2 based on a modified definition due to the special anisotropy of graphite. An approximate scheme is presented in Sec. II B 3 for efficient integration over the anisotropic first Brillouin zone (BZ) of graphite. Finally, in Sec. II B 4, the $a b$ initio phonon properties are verified by calculating the bulk thermal conductivity of graphite.

\section{First-principles calculation}

The first-principles calculation of graphite is implemented in the open-source package QUANTUM ESPRESSO (QE) [40]. The projected augmented wave pseudopotential with the Perdew-Burke-Ernzerhof exchange-correlation functional is adopted. To include the vdW interlayer interaction, the nonlocal vdW functional named optB88 is used as recommended by the previous studies [26,34]. Kinetic energy cutoffs of 60 and $400 \mathrm{Ry}$ are used for the wave function and charge density, respectively, and a convergence threshold of $10^{-12}$ is used for the self-consistent field calculation. Since graphite is semimetallic, the Marzari-Vanderbilt smearing [41] with a tiny Gaussian spreading of $0.02 \mathrm{Ry}$ is adopted for the electronic BZ integration. As a first step, the basal plane lattice constant and interlayer distance of graphite are obtained as $a=2.4646 \AA$ and $d=3.3364 \AA$ through a unit cell relaxation in QE with an electronic wave vector grid of $24 \times 24 \times 10$ and the convergence thresholds of $10^{-6}$ atomic units (a.u.), $10^{-4}$ a.u., and $0.05 \mathrm{kbar}$ for the total energy, force, and pressure, respectively. The values of lattice constants agree well with the widely accepted experimental data at low temperatures $(a=2.46 \AA$ and $d=3.34 \AA$ [42]). Then the harmonic force constants are computed by density functional perturbation theory in $\mathrm{QE}$ with an electronic wave vector grid of $24 \times 24 \times 12$ and a phonon q-mesh of

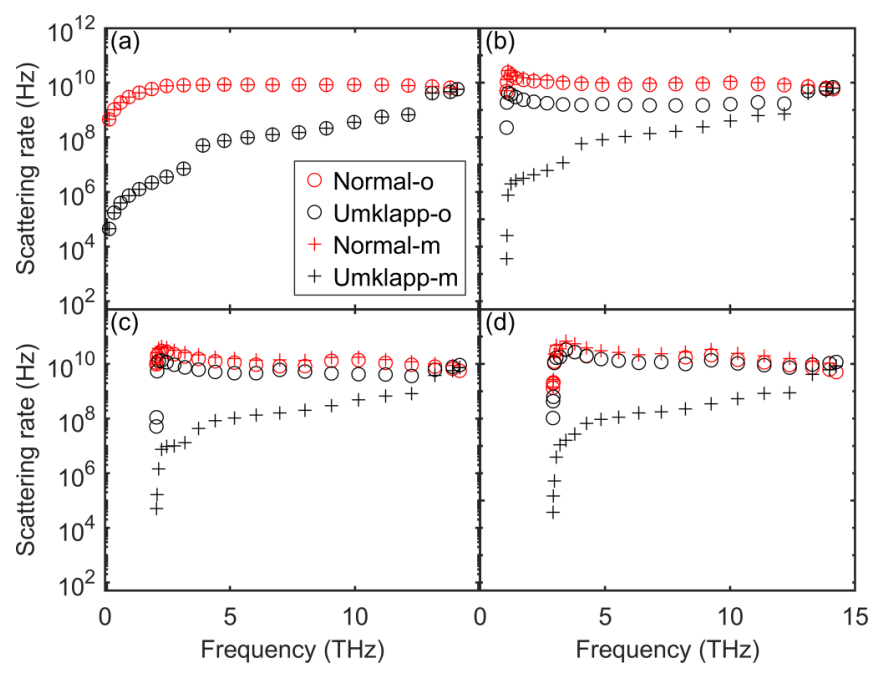

FIG. 1. Normal and Umklapp scattering rates of bending acoustic (BA) phonons in isotopically pure graphite at $100 \mathrm{~K}$ at different dimensionless $q_{z}$ from $\Gamma$ to $A$ points in the first Brillouin zone: (a) $Q_{z}=0$, (b) $Q_{z}=\frac{1}{6}$, (c) $Q_{z}=\frac{1}{3}$, and (d) $Q_{z}=\frac{1}{2}$. The red and black circles (or plus symbols) denote, respectively, the normal and umklapp scattering rates based on the original (-o) definition [or modified (-m) definition in Eq. (13)]. The representative results along the $\Gamma-M$ direction for $Q_{z}=0$ and along the directions parallel to $\Gamma-M$ within $\Gamma A L M$ for other $Q_{z}$ [c.f. Fig. 2(a)] are shown.

$5 \times 5 \times 2$. The third-order force constants are computed by the finite-displacement method implemented in the open-source script THIRDORDER [43]. A supercell of $4 \times 4 \times 2$ is adopted with the fourth nearest-neighbor atomic interaction included. The density functional theory calculation of atomic forces for different configurations is done in QE with an electronic wave vector grid of $4 \times$ $4 \times 4$. All the parameters in the first-principles calculation are chosen after a careful independence check.

\section{Modified definition of normal and Umklapp scattering rates}

With the harmonic and third-order force constants, the bulk phonon properties of graphite are computed in the opensource package SHENGBTE [43], with a phonon q-mesh of $40 \times 40 \times 6$ after an independence verification. The normal or Umklapp scattering rate is usually counted based on whether the phonon quasimomentum is conserved or not. Based on this original definition, the results at $100 \mathrm{~K}$ for the bending acoustic (BA) phonons at different dimensionless $q_{z}$ (denoted by $Q_{z}$ ) are shown as the circle points in Fig. 1. The Umklapp scattering is much weaker than the normal scattering in the low- and moderate-frequency ranges at $Q_{z}=0$, whereas it becomes stronger at larger $Q_{z}$ and even comparable to normal scattering at $Q_{z}=\frac{1}{2}$ (at the transverse edge of the first $\mathrm{BZ}$ ). This result seems to make sense since the phonon with a wave vector close to the transverse edge of the first $\mathrm{BZ}$ would easily go out of the first $\mathrm{BZ}$ when interacting with another phonon, i.e., an Umklapp scattering happens. However, it contradicts the results of phonon drift in graphite as detailed in Appendix A. The recent studies [26,35] proposed that the classification of normal and Umklapp scatterings in anisotropic materials should be based on the projected momentum along 


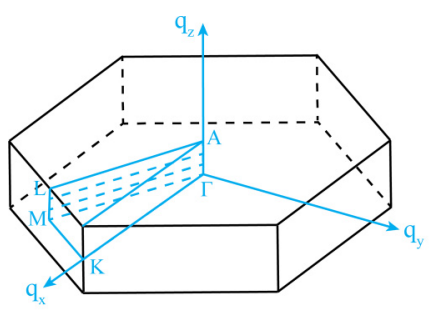

(a)

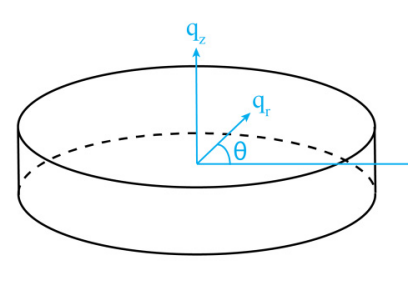

(b)
FIG. 2. Approximation of the first Brillouin zone (BZ) of graphite: (a) the realistic hexagonal first BZ and (b) the approximate cylindrical first BZ for solving Eq. (6). The dashed light blue lines within $\Gamma A L M$ in (a) denote the discrete directions parallel to $\Gamma-M$ at different $q_{z}$ from $\Gamma$ to $A$ points.

the heat flow direction. We adopt a slightly different directiondependent definition here. For heat transport along the basal plane of graphite, the normal or Umklapp scattering is counted based on whether the projected momentum along the basal plane is conserved or not in a three-phonon scattering:

$$
\mathbf{q}_{1, a b}+\mathbf{q}_{2, a b}=\mathbf{q}_{3, a b}+\mathbf{G}_{a b},
$$

where $\mathbf{G}$ is the reciprocal lattice vector, and the subscript $a b$ denotes the component along the basal plane ( $a b$ axis). The three-phonon scattering is a normal process if $\mathbf{G}_{a b}=0$, or an Umklapp process if $\mathbf{G}_{a b} \neq 0$. The modified definition is similar for heat transport along the $c$-axis direction. The present definition has one advantage over the previous one [26,35]: the normal and Umklapp scattering rates would be unique when the heat flow direction varies in real space along the basal plane of a graphite ribbon with irregular geometrical shapes. With the modified definition in Eq. (13), the trends of normal and Umklapp scattering rates shown by the plus symbols in Fig. 1 become consistent with the phonon drift results in Appendix A. Only the modified normal and Umklapp scattering rates based on Eq. (13) are calculated in this paper since we mainly focus on heat transport along the basal plane of graphite ribbon.

\section{Approximation of the first Brillouin zone}

The numerical solution of the space- and time-dependent phonon Boltzmann Eq. (6) would be computationally complicated and intensive if we directly integrate over the first BZ of graphite, as shown in Fig. 2(a). Here, we introduce an approximation of the first BZ by a cylindrical zone as shown in Fig. 2(b), where the phonon wave vector components $\left(q_{x}, q_{y}, q_{z}\right)$ turn into $\left(\theta, q_{r}, q_{z}\right)$. This approximate treatment makes the numerical integration very efficient since we could utilize the Gauss-Legendre (G-L) quadrature in the cylindrical coordinate, as introduced later in Sec. II C. Note that the first-principles calculation in Secs. II B 1 and II B 2 does not account for the approximations in this Sec. II B 3 . The obtained $a b$ initio phonon properties along the $\Gamma-M$ direction and the directions parallel to $\Gamma-M$ within $\Gamma A L M$ in the original first BZ in Fig. 2(a) are assumed, respectively, for $Q_{z}=0$ and for other $Q_{z}$ in the approximate first $\mathrm{BZ}$ in Fig. 2(b). Such an assumption is rather reasonable since the phonon properties along the $q_{x}-q_{y}$ plane at a specific $Q_{z}$ are
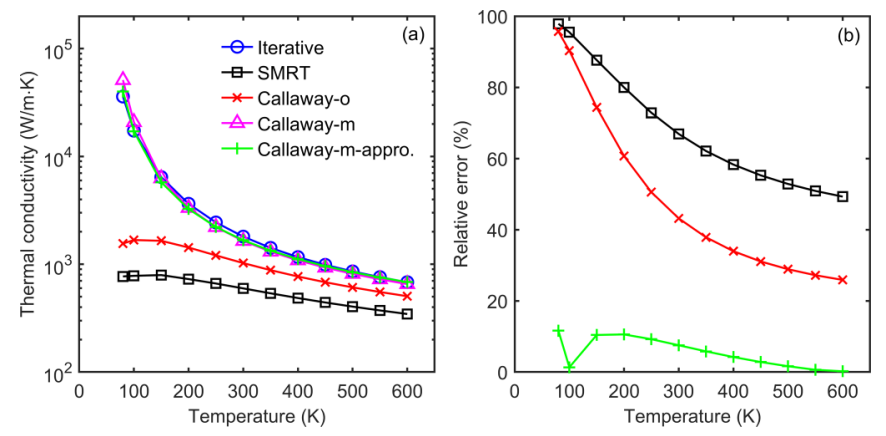

FIG. 3. Temperature-dependent bulk thermal conductivity of isotopically pure graphite: (a) basal plane result and (b) relative error of the relaxation approximations comparing with the iterative solution. The line with circles denotes the iterative solution of the phonon Boltzmann equation with a full scattering term; the line with squares denotes the single mode relaxation time (SMRT) approximation; the line with cross symbols (or triangles) denotes the Callaway's dual relaxation model with the original (-o) definition [or modified (-m) definition in Eq. (13)] of normal and Umklapp scattering rates, whereas the line with plus symbols denotes the Callaway's model with the modified definition of normal and Umklapp scattering rates under the approximations made in Sec. II B 3.

more or less isotropic. The present approximation is also applicable for other materials with a hexagonal first BZ, such as hexagonal BN [44], GaN, and AlN [45]. For materials with a complex first $\mathrm{BZ}$ where a good approximation is hard to find, we may have to integrate over the full first BZ in solving the phonon Boltzmann equation. As a last simplification, we only consider the six low-lying phonon branches (BA, TA, $\mathrm{LA}, \mathrm{BO}^{\prime}, \mathrm{TO}^{\prime}, \mathrm{LO}^{\prime}$ ) in the numerical solution of Eq. (6). The phonon dispersions of those branches at different $Q_{z}$ are illustrated in Appendix B. The contribution of the other six optical phonon branches to steady-state heat transport in this paper is much smaller and not considered for reducing the computational cost by half. As demonstrated in the recent work [26], even the $\mathrm{BA}, \mathrm{BO}^{\prime}$, and TA branches contribute to $>90 \%$ of the total thermal conductivity at $100 \mathrm{~K}$ where the hydrodynamic transport is relevant. The present consideration of six branches ensures a very good approximation in a broad range of temperatures, as to be shown in the following Subsec. II B 4. Technically speaking, all the phonon branches could be considered when necessary, for instance, in transient heat transport where all of them may significantly contribute to the heat capacity. Note that the effect of the omitted optical phonon branches is still included in the scattering rates of the considered branches through the perturbation theory calculation in the original full first BZ with all the phonon branches considered in Sec. II B 2.

\section{Bulk thermal conductivity calculation}

The ab initio force constants and phonon properties are verified by computing the bulk thermal conductivity of graphite, as reported in Fig. 3 for the isotopically pure $\left(0 \%{ }^{13} \mathrm{C}\right)$ case. The result of the natural abundance $\left(1.1 \%{ }^{13} \mathrm{C}\right)$ case is provided in Appendix $\mathrm{C}$ with a comparison with available experimental data. The iterative solution of the phonon Boltzmann equation with a full scattering term is obtained in 
SHENGBTE with the $a b$ initio force constants as input, whereas the results of the SMRT and Callaway's models are obtained with the phonon properties extracted from SHENGBTE. Both the SMRT model and Callaway's model with the original definition of normal and Umklapp scatterings appreciably underestimate the basal plane thermal conductivity, as shown in Fig. 3(a). The underestimation increases with decreasing temperature and reaches $\sim 100 \%$ at $\sim 100 \mathrm{~K}$, as given in Fig. 3(b). In contrast, with the modified definition of normal and Umklapp scattering rates in Sec. II B 2, Callaway's model shows an overall good agreement with the iterative solution. Furthermore, under the approximations in Sec. II B 3, i.e., based on a cylindrical first BZ with only six phonon branches considered, Callaway's model with the modified definition still agrees with the full iterative solution within $20 \%$, as quantified in Fig. 3(b). Callaway's model [21] has also been shown to agree with the iterative solution within $20-30 \%$ for the lattice thermal conductivity of diamond between 100 and $500 \mathrm{~K}$, covering the regime with comparable normal and Umklapp scatterings [22]. Despite sacrificing some accuracy, a direct numerical solution of Callaway's relaxation model is more efficient and feasible, while it remains an open challenge to solve the space-dependent phonon Boltzmann equation with a full scattering term in $3 \mathrm{D}$ materials. To sum up, the results demonstrate that the modified Callaway's model with (i) $a b$ initio phonon properties and (ii) the approximate treatment of the first BZ represents a good theoretical description of hydrodynamic heat transport along the basal plane of graphite.

\section{DOM}

In this subsection, we introduce a DOM for the direct numerical solution of the phonon Boltzmann Eq. (6) in the approximate cylindrical first BZ shown in Fig. 2(b). The steady-state heat transport along the basal plane of graphite ribbon is considered. Due to the weak interlayer vdW interaction, the size effect along the $c$ axis (thickness direction) of graphite is usually very small. As demonstrated in previous first-principles calculations [46] and experimental measurements [47], the thermal conductivity of multilayer graphene converges to that of bulk graphite as the number of atomic layers increases to $>\sim 5$. Thus, the $c$-axis direction could be in principle treated as bulk (i.e., periodic) if the thickness of graphite ribbon is larger than few nanometers, which is often the case in experiments [12] and in the focus of this paper. In other words, we consider 2D heat transport along the 3D graphite ribbon, where the size effect only comes from the boundary along the basal plane direction. The modeling of phonon hydrodynamics in a very thin graphite ribbon (or few-layer-graphene ribbon) with considerable size effect in the $c$ axis is more complicated and pending in a future work. Therefore, Eq. (6) is reduced to

$$
\mu \frac{\partial \phi}{\partial x}+\eta \frac{\partial \phi}{\partial y}=\frac{\phi_{\mathrm{R}}^{\mathrm{eq}}\left(T_{\mathrm{loc}, \mathrm{R}}\right)-\phi}{\Lambda_{\mathrm{R}}}+\frac{\phi_{\mathrm{N}}^{\mathrm{eq}}\left(T_{\mathrm{loc}, \mathrm{N}}, \mathbf{u}\right)-\phi}{\Lambda_{\mathrm{N}}}
$$

where $\mu=\cos \theta, \eta=\sin \theta$, and the basal plane mean free paths (MFPs) of the resistive and normal scatterings are calculated as $\Lambda_{\mathrm{R}}\left(q_{r}, q_{z}, p\right)=v_{g r} \tau_{\mathrm{R}}, \Lambda_{\mathrm{N}}\left(q_{r}, q_{z}, p\right)=v_{g r} \tau_{\mathrm{N}}$, with the basal plane group velocity defined as $v_{g r}\left(q_{r}, q_{z}, p\right)=$ $\sqrt{v_{g x}^{2}+v_{g y}^{2}}$.

The basic idea of the DOM scheme is to discretize the phonon distribution function on the real-space and reciprocalspace coordinates, with the details given in Appendix D. Here, we present the discrete integration scheme for the following general integral over the first BZ:

$$
I=\sum_{p} \int g(\mathbf{q}, p) \frac{d \mathbf{q}}{(2 \pi)^{3}}
$$

where $g(\mathbf{q}, p)$ is an arbitrary function of phonon mode. In the cylindrical coordinate system considered here, Eq. (15) is rewritten as

$$
I=\frac{\Delta q_{z}}{(2 \pi)^{3}} \sum_{p} \sum_{q_{z}} \int_{0}^{2 \pi} \int_{0}^{q_{r \max }} g\left(\theta, q_{r}, q_{z}, p\right) q_{r} d \theta d q_{r},
$$

where we have used a uniform rectangular integration over $q_{z}$ with an interval of $\Delta q_{z}$, as consistent with the calculation of bulk thermal conductivity in Sec. II B 4. The maximal basal plane wave vector is denoted by $q_{r \text { max }}$, which corresponds to the wave vector value at the $M$ point, as shown in Fig. 2(a). The integrations over $\theta$ and $q_{r}$ in Eq. (16) are calculated by the G-L quadrature as

$$
I=F \sum_{p} \sum_{q_{z}} \sum_{k=1}^{N_{\theta}} \sum_{n=1}^{N_{q r}} g_{n}^{k}\left(q_{z}, p\right) q_{r}(n) w_{k} w_{n},
$$

where $w_{k}, w_{n}$ are weight coefficients, and the integration factor $F=\frac{\Delta q_{z}}{(2 \pi)^{3}} \frac{1}{4} \pi q_{r \text { max }}$. The DOM scheme shown in Appendix D is derived based on the discretization in Eq. (17). Note that, to ensure the symmetry of the discrete angular space, $\theta \in[0,2 \pi]$ is divided into $[0 \pi]$ and $[\pi 2 \pi]$, and for each, the G-L quadrature with an equal number of abscissae $N_{\theta} / 2$ is applied [30].

\section{Validations}

In this subsection, we demonstrate a validation of the DOM scheme for the phonon Boltzmann equation under Callaway's model with the anisotropic $a b$ initio input. Heat transport along an infinitely long isotopically pure graphite ribbon with a finite width at 300 and $100 \mathrm{~K}$ is considered. A temperature gradient of $-10^{8} \mathrm{~K} / \mathrm{m}$ is exerted along the graphite ribbon. Such a case has been deeply investigated based on a semianalytical solution of Callaway's phonon Boltzmann equation in recent work [26]. This case is modeled by the present DOM solution with a grid of $N_{x}=2, N_{y}=201$ (301), $N_{\theta}=$ 64, $N_{q r}=10$ after independence verification. Note that $N_{p}=$ 6 and $N_{q z}=6$ are used for all the numerical solutions throughout this paper. The periodic heat flux boundary condition $[30,48]$ is used to implement the constant temperature gradient along the transport direction, whereas the diffuse scheme is used for the transverse adiabatic boundary [30,31]. The details of the boundary treatment are provided in Appendix D. We obtain the cross-sectional heat flux distributions along the graphite ribbons with various widths, which show an excellent agreement with the semi-analytical solutions [26] in Figs. 4(a) and 5(a). As a result, the widthdependent thermal conductivity of the graphite ribbon is also 

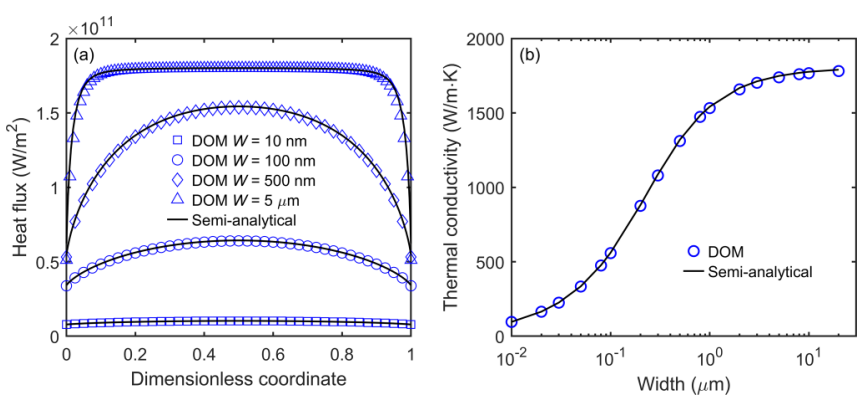

FIG. 4. Heat transport along an infinitely long graphite ribbon with a finite width at $300 \mathrm{~K}$ : (a) cross-sectional heat flux profile at different widths $(W)$ and (b) width-dependent thermal conductivity. The discrete symbols denote the present discrete-ordinate method (DOM) solution, whereas the solid lines denote the semi-analytical solution from Ref. [26]. Isotopically pure graphite is considered, and a temperature gradient of $-10^{8} \mathrm{~K} / \mathrm{m}$ is exerted.

well consistent with the semi-analytical result [26], as shown in Figs. 4(b) and 5(b). This benchmark study thus indicates the validation of the present DOM scheme. In the following Sec. III, we apply our computational framework to investigate heat transport in graphite ribbons with finite length and width, which would be no longer doable by semi-analytical solutions.

\section{RESULTS AND DISCUSSIONS}

In this section, the methodology developed in Sec. II is applied to model the classical in-plane and cross-plane heat transports along infinitely long and infinitely wide graphite ribbons, respectively, in Sec. III A. The size scaling of inplane and cross-plane thermal conductivities are revealed by the direct numerical solution and analyzed by a macroscopic hydrodynamic approach. In Sec. III B, the end effect on phonon Knudsen minimum in a graphite ribbon with a finite length and width is studied. An effective MFP model combining the size scaling of in-plane and cross-plane transports is introduced to interpret the results. For all cases, the isotopi-
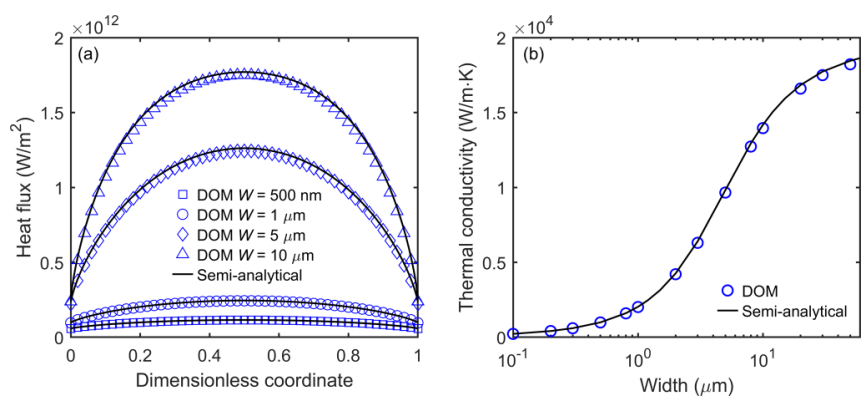

FIG. 5. Heat transport along an infinitely long graphite ribbon with a finite width at $100 \mathrm{~K}$ : (a) cross-sectional heat flux profile at different widths $(W)$ and (b) width-dependent thermal conductivity. The discrete symbols denote the present discrete-ordinate method (DOM) solution, whereas the solid lines denote the semi-analytical solution from Ref. [26]. Isotopically pure graphite is considered, and a temperature gradient of $-10^{8} \mathrm{~K} / \mathrm{m}$ is exerted.

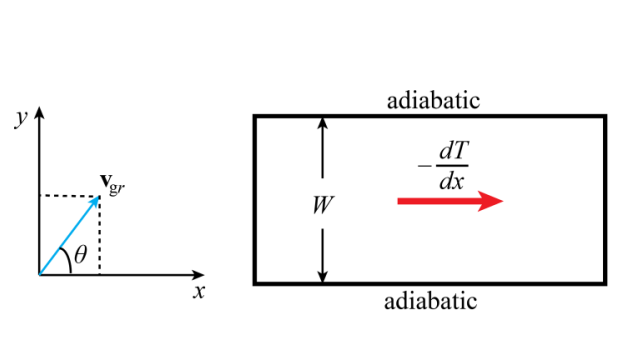

(a)

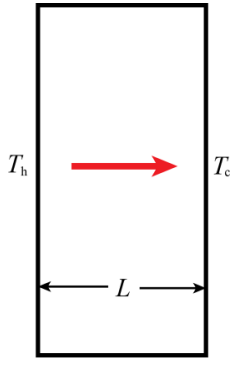

(b)
FIG. 6. Schematics of heat transport in the basal plane of the graphite ribbon: (a) in-plane heat transport along an infinitely long graphite ribbon with a finite width $W$ and (b) cross-plane heat transport along an infinitely wide graphite ribbon with a finite length $L$.

cally pure graphite is considered, which avoids the resistive isotope scattering to deteriorate the hydrodynamic effect.

\section{A. In-plane and cross-plane heat transports}

In this subsection, we model the in-plane heat transport along an infinitely long graphite ribbon with a finite width $W$ in Fig. 6(a) and the cross-plane heat transport along an infinitely wide graphite ribbon with a finite length $L$ in Fig. 6(b). Both the in-plane and cross-plane transports are along the basal plane of graphite. Three different average system temperatures are considered: $T_{0}=80,100$, and $300 \mathrm{~K}$, which covers both the hydrodynamic regime ( 80 and $100 \mathrm{~K})$ and the diffusive regime $(300 \mathrm{~K})$. A constant temperature gradient is implemented along the transport direction of the in-plane case, whereas hot and cold sources are exerted with a tiny temperature difference of $1 \mathrm{~K}$ in the cross-plane case. The numerical grids and boundary treatment for the in-plane transport are the same as those in Sec. IID. For the cross-plane transport, the DOM in Sec. II C is reduced to a one-dimensional (1D) formulation, with a spatial grid of $N_{x}=201$ and $N_{x}=101$ adopted for $T_{0}=80$ and $100 \mathrm{~K}$ and $T_{0}=300 \mathrm{~K}$, respectively, whereas $N_{\theta}=32$ and $N_{q r}=10$ for all. The phonon blackbody isothermal boundary conditions [30,31] are used for the hot and cold sources.

\section{Size scaling of thermal conductivity}

The size-dependent in-plane and cross-plane thermal conductivities of graphite ribbons at different temperatures are demonstrated in Fig. 7. In all three cases, the in-plane thermal conductivity is higher than the cross-plane one at the same characteristic length (ribbon size), which is like the trend in the ballistic-diffusive heat transport through silicon thin films $[49,50]$. However, by decreasing temperature from 300 to $80 \mathrm{~K}$, the difference between in-plane and cross-plane thermal conductivities increases. Specifically, a considerably faster increase of in-plane thermal conductivity appears at intermediate ribbon size than the cross-plane one at 80 and $100 \mathrm{~K}$, as clearly seen in Figs. 7(a) and 7(b). Thus, the convergence of the thermal conductivity occurs at smaller size in the in-plane transport, for instance, at $\sim 50 \mu \mathrm{m}$ compared with $\sim 500 \mu \mathrm{m}$ in the cross-plane transport at $100 \mathrm{~K}$, as shown in Fig. 7(b). In other words, a much smaller characteristic length is required 

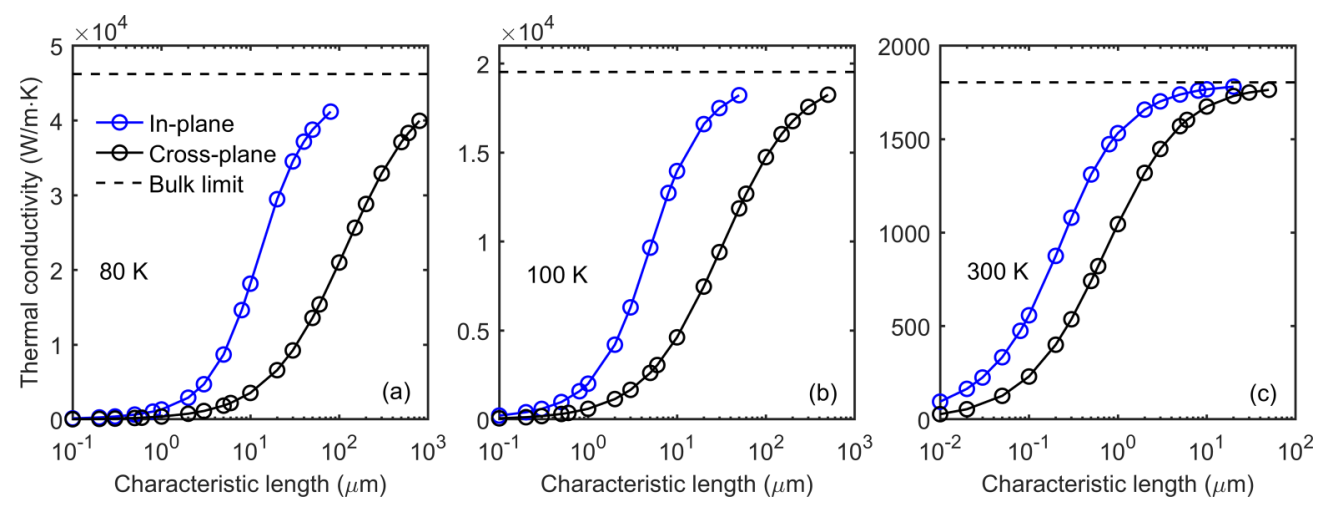

FIG. 7. Thermal conductivity of in-plane and cross-plane heat transport along infinitely long and infinitely wide graphite ribbons, respectively, at different temperatures: (a) $80 \mathrm{~K}$, (b) $100 \mathrm{~K}$, and (c) $300 \mathrm{~K}$. Isotopically pure graphite is considered here, and the characteristic length denotes the width for the in-plane case or the length for the cross-plane case (c.f. Fig. 6).

to see the size effect in the in-plane heat transport in the hydrodynamic regime at lower temperatures.

The very different size effects of the in-plane and crossplane heat transports come from the different size scaling behaviors, as given by the normalized thermal conductivity by the ribbon size in Fig. 8. The ribbon size is proportional to the thermal conductivity in the ballistic limit [36,51], where the phonon MFP is limited by the boundary of the graphite ribbon. The normalized cross-plane thermal conductivity always decreases with increasing ribbon size at different temperatures. The continual decrease with ribbon size is different from the trend of convergence after a critical size comparable to the phonon MFP in previous works $[28,32]$ with only the normal scattering considered. In contrast, the normalized thermal conductivity shows a nonmonotonic trend with the ribbon size in the in-plane transport at 80 and $100 \mathrm{~K}$, as shown in Figs. 8(a) and 8(b). For instance, at $80 \mathrm{~K}$ in Fig. 8(a), it even increases with ribbon size after a minimum at $\sim 800 \mathrm{~nm}$ and reaches a maximum at $\sim 8 \mu \mathrm{m}$ before decreasing again. The minimum is known as the phonon Knudsen minimum due to the transition from ballistic to hydrodynamic transports $[26,30,36]$. The increase with ribbon size between 0.8 and $8 \mu \mathrm{m}$ represents the super-ballistic scaling of in-plane heat transport in the hydrodynamic regime $[19,26]$. This explains the much faster increase of in-plane thermal conductivity than the cross-plane one in Figs. 7(a) and 7(b). The super-ballistic scaling with the transverse dimension in the presence of dominant normal scattering has been attributed to the effective momentum-destroying MFP $\Lambda_{\text {eff }} \sim W^{2} / \Lambda_{\mathrm{N}}$, which was deduced by a random walk theory [37,38]. In the following Sec. III A 2, we provide a unified analysis, based on a hydrodynamic approach, of the size scaling for the in-plane heat transport that is known and for the cross-plane transport that is less quantified.

\section{Size scaling analysis by hydrodynamic approach}

We adopt the following phonon hydrodynamic equation for our analysis:

$$
\tau_{\mathrm{R}} \frac{\partial \mathbf{J}}{\partial t}+\mathbf{J}=-\frac{1}{3} C_{V} v_{g}^{2} \tau_{\mathrm{R}} \nabla T+\frac{1}{5} \tau_{\mathrm{N}} \tau_{\mathrm{R}} v_{g}^{2}\left[\nabla^{2} \mathbf{J}+\frac{1}{3} \nabla(\nabla \cdot \mathbf{J})\right]
$$

Equation (18) can be derived from the phonon Boltzmann equation under Callaway's dual relaxation model with two assumptions [5,23]: (i) gray phonon properties and (ii) dominant normal scattering over the resistive one $\left(\Lambda_{\mathrm{N}} \ll \Lambda_{\mathrm{R}}\right)$. It is actually a variant of the classical Guyer-Krumhansl heat
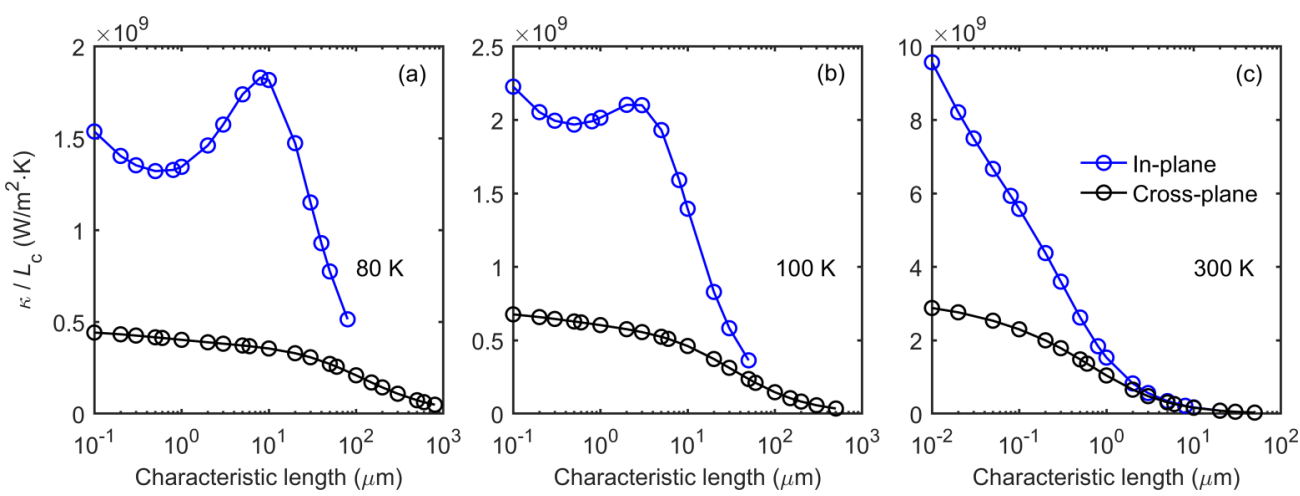

FIG. 8. Normalized thermal conductivity of in-plane and cross-plane heat transport along infinitely long and infinitely wide graphite ribbons, respectively, at different temperatures: (a) $80 \mathrm{~K}$, (b) $100 \mathrm{~K}$, and (c) $300 \mathrm{~K}$. Isotopically pure graphite is considered here, and the characteristic length $\left(L_{c}\right)$ denotes the width for the in-plane case or the length for the cross-plane case (c.f. Fig. 6). The thermal conductivity normalized by the characteristic length denotes a normalization by the ballistic limit. 
equation [4,52] with a minor coefficient difference in the nonlocal terms of the heat flux [5,23]. To model the in-plane and cross-plane heat transports, we also need the boundary conditions of heat flux slip and temperature jump, respectively $[23,53]$ :

$$
\begin{gathered}
\mathbf{J}_{\mathrm{s}}=\frac{8}{15} \Lambda_{\mathrm{N}}\left(\frac{\partial \mathbf{J}}{\partial n}\right)_{s}, \\
T_{s}-T=-\frac{2}{3} \Lambda_{\mathrm{R}}\left(\frac{\partial T}{\partial n}\right)_{s},
\end{gathered}
$$

where the subscript $s$ denotes the boundary, and $n$ in the partial derivative represents the normal direction of the boundary. Equation (20) indicates that the cross-plane temperature jump near the heat source is only related to the resistive scattering. Physically speaking, this is because the phonon viscous effect via normal scattering only emerges in the presence of a transverse boundary, as in the in-plane heat transport. In other words, cross-plane heat transport in the hydrodynamic regime resembles the inviscid flow in fluid mechanics [54]. From the mathematical point of view, the nonlocal terms of heat flux on the right-hand side of Eq. (18) (similar to the viscous terms in Navier-Stokes hydrodynamic equation [54]) will vanish and have no contribution to the 1D cross-plane heat transport. The phonon hydrodynamic Eq. (18) together with the boundary conditions Eqs. (19) and (20) have been validated through a comparison with the direct DOM solution of Callaway's phonon Boltzmann equation [53].

For the steady-state in-plane heat transport, we obtain the heat flux solution of Eq. (18) with the boundary condition in Eq. (19) and then compute the effective thermal conductivity as follows [53]:

$$
\frac{\kappa_{\mathrm{eff}}}{\kappa}=1-\frac{2 K n_{m} \tanh \left(\frac{1}{2 K n_{m}}\right)}{1+\frac{8}{15} \frac{K n_{\mathrm{N}}}{K n_{m}} \tanh \left(\frac{1}{2 K n_{m}}\right)},
$$

where $\kappa=\frac{1}{3} C_{V} v_{g} \Lambda_{\mathrm{R}}, K n_{\mathrm{m}}=\sqrt{K n_{\mathrm{N}} K n_{\mathrm{R}} / 5}$, with the Knudsen numbers for normal and resistive scatterings defined as $K n_{\mathrm{N}}=\Lambda_{\mathrm{N}} / W, K n_{\mathrm{R}}=\Lambda_{\mathrm{R}} / W$. In the phonon hydrodynamic regime (or phonon Poiseuille flow regime) we are concerned with, the window condition applies as [4] $\Lambda_{\mathrm{N}} \ll W, \Lambda_{\mathrm{N}} \Lambda_{\mathrm{R}} \gg$ $W^{2}$, which gives rise to $K n_{\mathrm{N}} \ll 1 \ll K n_{m} \ll K n_{\mathrm{R}}$. Therefore, Eq. (21) can be approximated as

$$
\begin{aligned}
\frac{\kappa_{\mathrm{eff}}}{\kappa} & \simeq 1-2 K n_{m} \tanh \left(\frac{1}{2 K n_{m}}\right) \\
& \simeq 1-2 K n_{m}\left[\frac{1}{2 K n_{m}}-\frac{1}{3}\left(\frac{1}{2 K n_{m}}\right)^{3}\right] \\
& =\frac{1}{12 K n_{m}^{2}} .
\end{aligned}
$$

With the expressions of $\kappa$ and $K n_{m}$, Eq. (22) yields the effective thermal conductivity as $\kappa_{\text {eff }}=\frac{1}{3} C_{V} v_{g}\left(\frac{5}{12} \frac{W^{2}}{\Lambda_{\mathrm{N}}}\right)$. Using the classical kinetic formula of thermal conductivity, we obtain an effective momentum-destroying MFP $\Lambda_{\text {eff }}=\frac{5}{12} \frac{W^{2}}{\Lambda_{\mathrm{N}}}$, which is well consistent with the size scaling of $\Lambda_{\text {eff }} \sim W^{2} / \Lambda_{\mathrm{N}}$ based on the random walk theory $[37,38]$.

For the steady-state cross-plane heat transport, we obtain the solution of Eq. (18) with the boundary condition in Eq. (20) and then compute the effective thermal conductivity as follows [53]:

$$
\frac{\kappa_{\mathrm{eff}}}{\kappa}=\frac{1}{1+\frac{4}{3} K n_{\mathrm{R}}},
$$

where $K n_{\mathrm{R}}=\Lambda_{\mathrm{R}} / L$ here. Using the kinetic formula of thermal conductivity, we obtain the effective momentumdestroying MFP from Eq. (23) as

$$
\frac{1}{\Lambda_{\mathrm{eff}}}=\frac{1}{\Lambda_{\mathrm{R}}}+\frac{4}{3} \frac{1}{L} .
$$

Equation (24) is like the Mathiessen's rule and shows that the cross-plane thermal resistance combines the first term $\left(1 / \Lambda_{R}\right)$ from the resistive scattering and the second term from the heat source boundaries $(\sim 1 / L)$. In the phonon hydrodynamic regime here, $\Lambda_{\mathrm{R}} \gg L$, which means that the second term is dominant. The size scaling for cross-plane heat transport thus is written as $\Lambda_{\text {eff }} \sim L$ and $\kappa_{\text {eff }} / L \sim C_{V} v_{g} / 4$. The result based on the present analysis agrees well with the trends in Figs. 8(a) and $8(\mathrm{~b})$, where $\kappa / L_{c}$ is weakly dependent on $L_{c}$ within the range of the hydrodynamic regime (e.g., the corresponding range of ribbon size from the minimum to the maximum of the in-plane case). A recent study [32] in the hypothetical graphitic materials with constant normal scattering rate also showed an invariable thermal resistance when $\Lambda_{\mathrm{N}}<L$, i.e., in the hydrodynamic regime. Actually Eqs. (23) and (24) are valid from the hydrodynamic regime $\left(\Lambda_{\mathrm{R}} \gg L\right)$ to the diffusive regime $\left(\Lambda_{\mathrm{R}} \ll L\right)$, as verified previously [53]. They yield the following scaling for the cross-plane thermal conductivity:

$$
\frac{\kappa_{\mathrm{eff}}}{L}=\frac{1}{3} C_{V} v_{g} \frac{\Lambda_{\mathrm{R}}}{L+\frac{4}{3} \Lambda_{\mathrm{R}}},
$$

which indeed captures the general trend in Figs. 8(a) and 8(b) in the whole range of $L_{c}$. It is also interesting to find that Eqs. (23)-(25) are like the classical results for the transition from ballistic to diffusive transports across a thin film [55]. This explains the similarity of the hydrodynamic-to-diffusive transition at 80 and $100 \mathrm{~K}$ in Figs. 8(a) and 8(b) to the ballistic-to-diffusive transition in Fig. 8(c) at $300 \mathrm{~K}$ for the cross-plane case. It means that the viscous effect of normal scattering is almost invisible in the cross-plane heat transport as we mentioned precedingly.

To provide an intuitive picture of the dominant thermal resistance from the heat source boundaries in the hydrodynamic regime, we display the temperature distributions for the cross-plane heat transport in Fig. 9. In the range of the hydrodynamic regime [e.g., $L=5 \mu \mathrm{m}$ at $80 \mathrm{~K}$ in Fig. 9(a) and $L=1 \mu \mathrm{m}$ at $100 \mathrm{~K}$ in Fig. 9(b)], the temperature profile is very uniform inside the graphite ribbon, whereas very large temperature jumps near both heat sources occur. Furthermore, a stronger nonlinearity of the temperature profile appears near the boundary in the hydrodynamic regime than that in the diffusive regime [i.e., at $300 \mathrm{~K}$ in Fig. 9(c)], which is attributed to the large mismatch between the displaced phonon distribution inside the ribbon and the isotropic Bose-Einstein distribution in the heat source [28,32].

To sum up, we obtain the size scaling of $\Lambda_{\text {eff }} \sim W^{2} / \Lambda_{\mathrm{N}}$ and $\Lambda_{\text {eff }} \sim L$, respectively, for in-plane and cross-plane heat transports in the hydrodynamic regime based on a phonon 

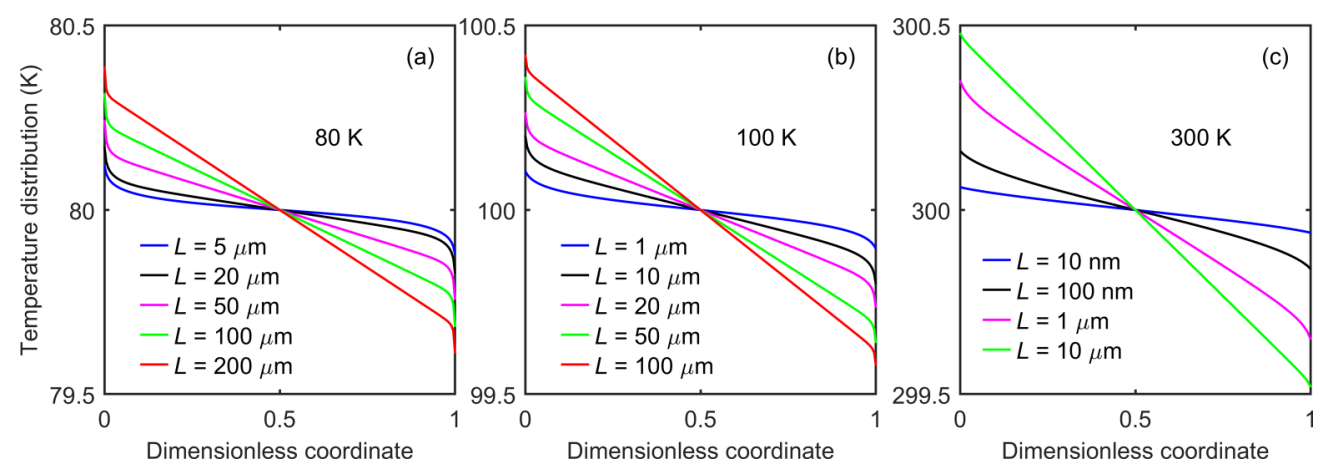

FIG. 9. Temperature distribution of cross-plane heat transport along an infinitely wide graphite ribbon with different lengths $(L)$ at several temperatures: (a) $80 \mathrm{~K}$, (b) $100 \mathrm{~K}$, and (c) $300 \mathrm{~K}$. Isotopically pure graphite is considered here.

hydrodynamic approach. The present analysis provides a good interpretation of the results in Sec. III A 1 and will also help us to understand the end effect on the phonon Knudsen minimum in the following Sec. III B.

\section{B. End effect on phonon Knudsen minimum}

The phonon Knudsen minimum has appeared in the inplane heat transport along an infinitely long graphite ribbon at 80 and $100 \mathrm{~K}$ in Sec. III A. In this subsection, we investigate the end effect on the phonon Knudsen minimum in a rectangular graphite ribbon with finite length and width at 100 and $80 \mathrm{~K}$, as shown in Fig. 10. For the first case at $100 \mathrm{~K}$, a series of ribbon lengths of $10,20,30,50$, and $80 \mu \mathrm{m}$ is considered, and for each one, the ribbon width varies from $100 \mathrm{~nm}$ to 50 $\mu \mathrm{m}$ to study the width dependence of heat transport. A spatial grid of $N_{x}=101$ and $N_{y}=101$ is adopted after an independence check. For the second case at $80 \mathrm{~K}$, a series of ribbons lengths of $10,20,30,50$, and $100 \mu \mathrm{m}$ is considered; for each one, the same range of width from $100 \mathrm{~nm}$ to $50 \mu \mathrm{m}$ is studied. A spatial grid of $N_{x}=101$ and $N_{y}=51$ is adopted for $W \leqslant$ $500 \mathrm{~nm}$, whereas a spatial grid of $N_{x}=101$ and $N_{y}=101$ is used for other widths after an independence check. For both cases at 100 and $80 \mathrm{~K}, N_{\theta}=48, N_{q r}=10$ are considered after an independence check. A tiny temperature difference of $1 \mathrm{~K}$ is implemented on the graphite ribbon. The isothermal and adiabatic boundary treatments are the same as those in Sec. III A.

The width-dependent thermal conductivity and its normalization over the ballistic limit (proportional to the width $W$ ) at different ribbon lengths are given in Figs. 11 and 12 for the cases of 100 and $80 \mathrm{~K}$, respectively. The results for an infinitely long graphite ribbon are also included as a reference,
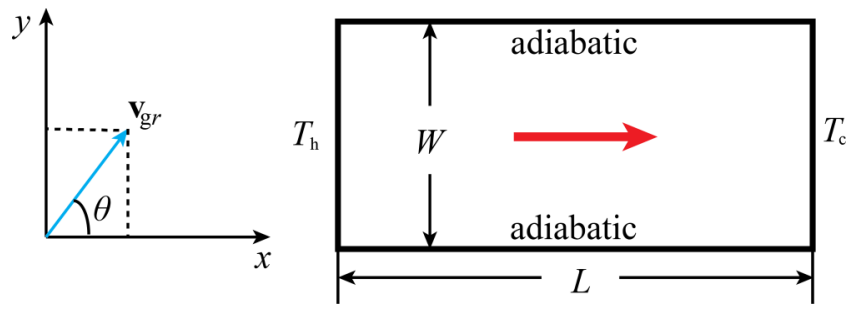

FIG. 10. Schematic of heat transport along a graphite ribbon with a finite length $L$ and a finite width $W$. where a Knudsen minimum is clearly seen around the lower limit of the ribbon width range in the hydrodynamic regime (from $\sim 500 \mathrm{~nm}$ to $\sim 3 \mu \mathrm{m}$ at $100 \mathrm{~K}$ and from $\sim 800 \mathrm{~nm}$ to $\sim 8 \mu \mathrm{m}$ at $80 \mathrm{~K}$ ). However, the minimum disappears when the ribbon length lies also within the similar range, for instance, at $10 \mu \mathrm{m}$. At both temperatures, the thermal conductivity of the graphite ribbon with a length of $10 \mu \mathrm{m}$ converges very fast with width to a saturation value much smaller than the bulk limit, which implies a very strong size effect from the finite length. The ribbon length where the Knudsen minimum reemerges is $>\sim 50 \mu \mathrm{m}$ at both 100 and $80 \mathrm{~K}$, i.e., much larger than the width range that supports hydrodynamic transport. Also, the minimum in the graphite ribbon with $L=80 \mu \mathrm{m}$ at $100 \mathrm{~K}$ is comparatively milder than that in the ribbon with $L=100 \mu \mathrm{m}$ at $80 \mathrm{~K}$, due to the relatively stronger Umklapp scattering and weaker hydrodynamic effect in the former case. Because of the strong end effect, a critical ribbon length to observe the phonon Knudsen minimum shall be $\sim 5-10$ times the upper limit of the width range in the hydrodynamic regime. This critical ribbon length is dependent on the system temperature and could be estimated based on the temperaturedependent result of the width range in the hydrodynamic regime [26]. A similar critical length-to-height ratio of 5-10 has been found in the Knudsen minimum of gas flow in a planar microchannel with finite length [56,57]. To illustrate the end effect, we also display the middle cross-sectional heat flux profiles inside the graphite ribbon with $W=2 \mu \mathrm{m}$ at $100 \mathrm{~K}$ and $W=5 \mu \mathrm{m}$ at $80 \mathrm{~K}$ at different lengths, as shown
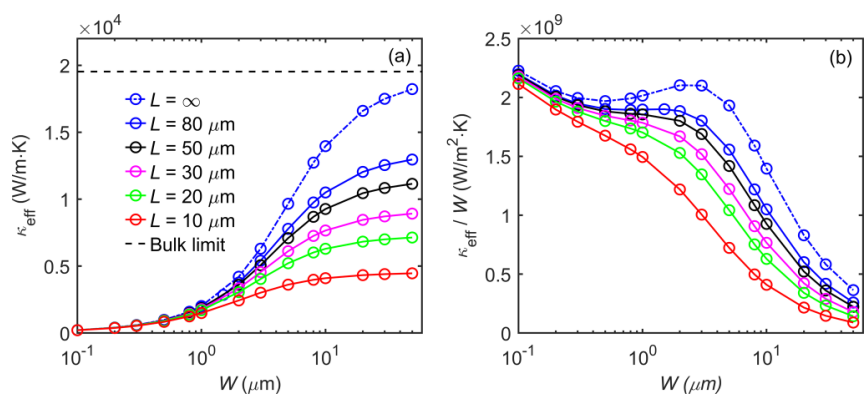

FIG. 11. (a) Width-dependent thermal conductivity and (b) normalized thermal conductivity of rectangular graphite ribbons with different lengths $(L)$ at $100 \mathrm{~K}$. Isotopically pure graphite is considered here, and the thermal conductivity normalized by the width $(W)$ in (b) denotes the normalization by the ballistic limit. 

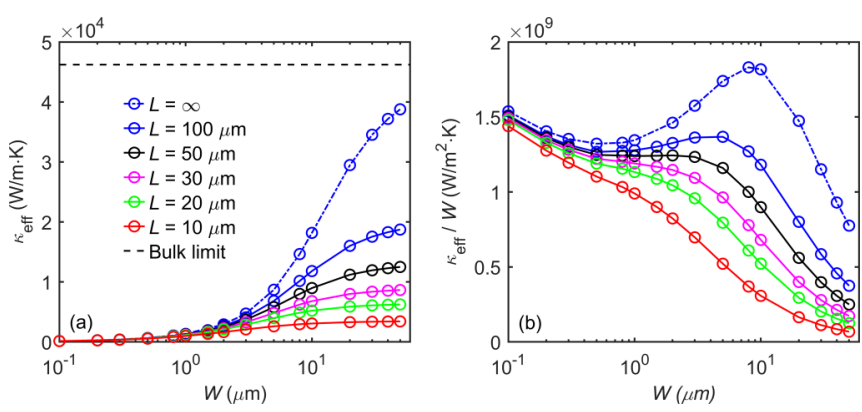

FIG. 12. (a) Width-dependent thermal conductivity and (b) normalized thermal conductivity of rectangular graphite ribbons with different lengths $(L)$ at $80 \mathrm{~K}$. Isotopically pure graphite is considered here, and the thermal conductivity normalized by the width $(W)$ in (b) denotes the normalization by the ballistic limit.

in Figs. 13(a) and 13(b), respectively. The results indicate that the finite ribbon length generates a significant restriction on the development of the hydrodynamic heat flow.

To establish an intuitive understanding of the strong end effect on the phonon Knudsen minimum, we provide an interpretation via a MFP analysis. The effective phonon MFP in a rectangular graphite ribbon can be estimated by the Mathiessen's law as

$$
\frac{1}{\Lambda_{\mathrm{eff}}}=\frac{1}{\Lambda_{\mathrm{eff}, L}}+\frac{1}{\Lambda_{\mathrm{eff}, W}},
$$

where $\Lambda_{\text {eff, } L}$ and $\Lambda_{\text {eff, } W}$ correspond to the effective momentum-destroying MFP in the length and width directions, respectively. Based on the preceding analysis of Sec. III A 2, the effective momentum-destroying MFPs in the hydrodynamic regime reads

$$
\Lambda_{\text {eff, } W} \sim \frac{W^{2}}{\Lambda_{\mathrm{N}}}, \quad \Lambda_{\text {eff, } L} \sim L .
$$

Originated from the super-ballistic scaling in the width direction, the Knudsen minimum would take place only when $\Lambda_{\text {eff, } W}<\Lambda_{\text {eff, } L}$, i.e., the phonon quasimomentum is preserved in the width direction before it is destroyed in the length direction. Thus, $L>W^{2} / \Lambda_{\mathrm{N}}$, which implies $L \gg W$ since $W / \Lambda_{\mathrm{N}} \gg 1$ in the hydrodynamic regime. Consequently, the length shall be much larger than the width to observe the phonon Knudsen minimum in a rectangular graphite ribbon, which is consistent with the results of our direct numerical solutions. Although the present effective MFP analysis is based on a gray phonon hydrodynamic model, it captures the crucial point and predicts the correct general trend. This paper thus also demonstrates the power of macroscopic hydrodynamic equations [5] in understanding non-Fourier heat transport, which have followed appreciable progress in recent years $[6-9,58]$.

\section{CONCLUSIONS}

In summary, we investigated the phonon hydrodynamics in graphite micro- and nanostructures based on a direct numerical solution of the phonon Boltzmann equation under Callaway's model with input from the first-principles calculations of anisotropic phonon properties. In the hydrodynamic regime, the effective momentum-destroying phonon MFPs in the transverse and longitudinal directions are proportional to the width square and the length of the system, respectively. A strong end effect is thus uncovered in the phonon Knudsen minimum along finite-sized graphite ribbon, which occurs only when the ribbon length is much larger than the width. This paper provides an efficient and robust computational framework for the theoretical modeling and experimental investigation of hydrodynamic heat transport in anisotropic materials in the future. Our theoretical analysis also promotes the understanding of the size effect on thermal conductivity in the phonon hydrodynamic regime. During the peer review process, we noticed a report of a Monte Carlo solution of the time- and space-dependent phonon Boltzmann equation with a full scattering matrix in a circular graphite ribbon [59], which is generally more computationally intensive.

\section{ACKNOWLEDGMENTS}

This paper was supported by the Postdoctoral Fellowship of Japan Society for the Promotion of Science (No. P19353), and CREST Japan Science and Technology Agency (No. JPMJCR19I1 and No. JPMJCR19Q3). This research used the
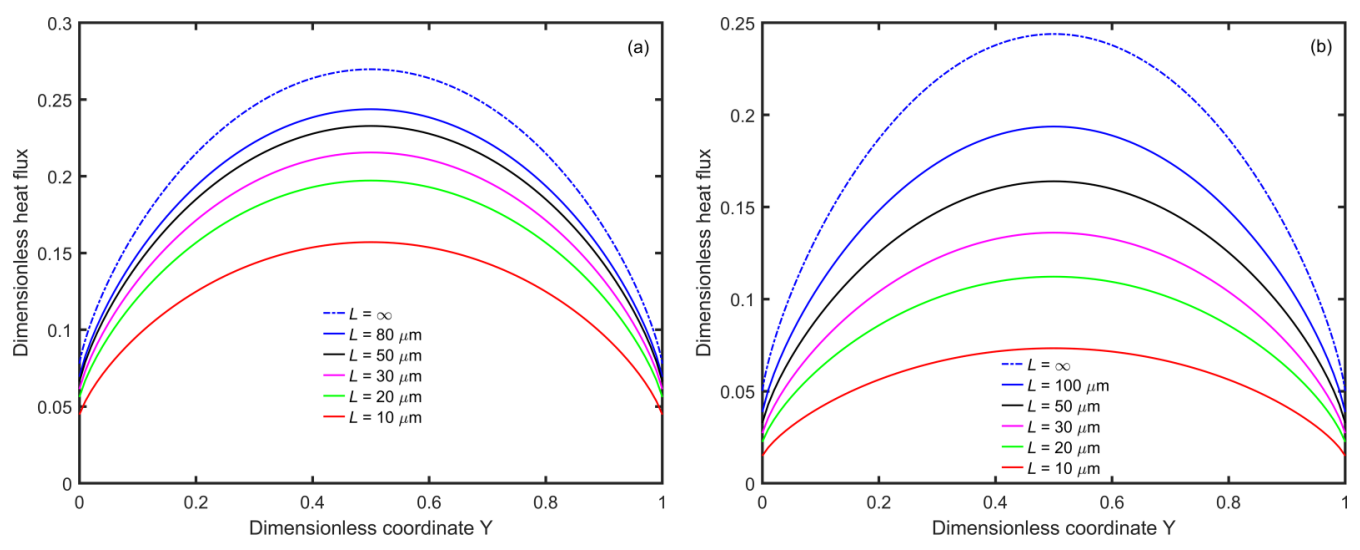

FIG. 13. The middle cross-sectional dimensionless heat flux profile of heat transport in rectangular graphite ribbons with different lengths (L): (a) $W=2 \mu \mathrm{m}, 100 \mathrm{~K}$; and (b) $W=5 \mu \mathrm{m}, 80 \mathrm{~K}$. Isotopically pure graphite is considered here, and the heat flux is normalized by the bulk limit. 
computational resources of the Oakforest-PACS supercomputer system, The University of Tokyo.

\section{APPENDIX A: PHONON DRIFT IN GRAPHITE}

The phonon drift is characterized by the normalized deviation of the distribution function defined as [11] $d \bar{f}=$ $\left(f_{\mathbf{q} p}-f_{0}\right) /\left[f_{0}\left(f_{0}+1\right)\right]$, with $f_{0}$ the Bose-Einstein distribution and $f_{\mathbf{q} p}$ obtained through an iterative solution of the phonon Boltzmann equation in SHENGBTE. As shown in Fig. 14, the phonon drift along the basal plane of graphite is almost independent of $Q_{z}$. In other words, the normal scattering is still much stronger than the Umklapp one, even near the transverse edge of the first BZ. This contradicts the results of normal and Umklapp scattering rates based on the original definition in Fig. 1. Such a contradiction indicates that the usual definition of normal and Umklapp scatterings does not work well for graphite as a strongly anisotropic system.

\section{APPENDIX B: PHONON DISPERSION OF GRAPHITE}

The phonon dispersion of the six low-lying branches of graphite is demonstrated in Fig. 15.

\section{APPENDIX C: EXPERIMENTAL VALIDATION OF $A B$ INITIO PHONON PROPERTIES OF GRAPHITE}

In the natural abundance case, the iterative solution and the modified Callaway's model generally agree well with the available experimental data of bulk thermal conductivity of

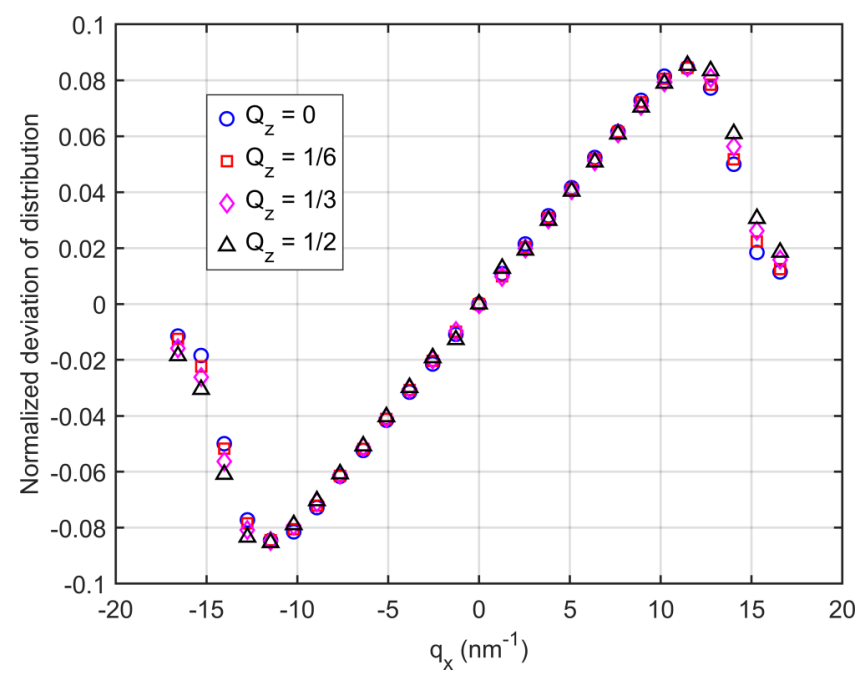

FIG. 14. Normalized deviation of distribution for bending acoustic (BA) phonons in isotopically pure graphite at $100 \mathrm{~K}$ at different dimensionless $q_{z}$ from $\Gamma$ to $A$ points in the first Brillouin zone. The results are obtained through an iterative solution of phonon Boltzmann equation with the full scattering term. A temperature gradient of $\left[-10^{8} 00\right](\mathrm{K} / \mathrm{m})$ is applied. The results at $q_{y}=0$ are shown.

graphite [60], as shown in Fig. 16(a). Also, the present iterative solution is quite consistent with the reference one [35] with the same pseudopotential and vdW functional, inferring that the first-principles calculation in Sec. II B 1 is reliable. For the $c$-axis thermal conductivity, both the SMRT model and the original Callaway's model reproduce well the iterative solution and experimental result, as shown in Fig. 16(b). This indicates weak hydrodynamic effect along the $c$-axis direction of graphite.

\section{APPENDIX D: DOM}

The details of the DOM are introduced for the phonon Boltzmann equation under Callaway's dual relaxation model with anisotropic phonon properties. With the same numerical discretization in Eq. (17), the discrete form of the phonon Boltzmann Eq. (14) in the wave vector space is formulated as

$$
\mu_{k} \frac{\partial \phi_{n}^{k}(l, p)}{\partial x}+\eta_{k} \frac{\partial \phi_{n}^{k}(l, p)}{\partial y}=\frac{\left(\phi_{\mathrm{R}}^{\mathrm{eq}}\right)_{n}(l, p)-\phi_{n}^{k}(l, p)}{\left(\Lambda_{\mathrm{R}}\right)_{n}(l, p)}+\frac{\left(\phi_{\mathrm{N}}^{\mathrm{eq}}\right)_{n}^{k}(l, p)-\phi_{n}^{k}(l, p)}{\left(\Lambda_{\mathrm{N}}\right)_{n}(l, p)}
$$

where $k=1,2, \ldots, N_{\theta}, n=1,2, \ldots, N_{q r}, l=1,2, \ldots, N_{q z}$, and $p=1,2, \ldots, N_{p}$ represent the index of discrete angular variables $(\theta)$, basal plane wave vectors $\left(q_{r}\right), c$-axis wave vectors $\left(q_{z}\right)$, and phonon polarization $(p)$. The discrete equilibrium distribution functions for the resistive and normal scatterings in Eq. (D1) can be derived from Eqs. (7) and (8) with the aid of Eq. (17):

$$
\begin{gathered}
\left(\phi_{\mathrm{R}}^{\mathrm{eq}}\right)_{n}(l, p)=\frac{C_{n}(l, p)}{C_{\tau_{\mathrm{R}}}} F \sum_{p^{\prime}} \sum_{l^{\prime}} \sum_{k^{\prime}=1}^{N_{\theta}} \sum_{n^{\prime}=1}^{N_{q r}} \frac{\phi_{n^{\prime}}^{k^{\prime}}\left(l^{\prime}, p^{\prime}\right)}{\left(\tau_{\mathrm{R}}\right)_{n^{\prime}}\left(l^{\prime}, p^{\prime}\right)} q_{r}\left(n^{\prime}\right) w_{k^{\prime}} w_{n^{\prime}}, \\
\left(\phi_{\mathrm{N}}^{\mathrm{eq}}\right)_{n}^{k}(l, p)=\frac{C_{n}(l, p)}{C_{\tau_{\mathrm{N}}}} F \sum_{p^{\prime}} \sum_{l^{\prime}} \sum_{k^{\prime}=1}^{N_{\theta}} \sum_{n^{\prime}=1}^{N_{q r}} \frac{\phi_{n^{\prime}}^{k^{\prime}}\left(l^{\prime}, p^{\prime}\right)}{\left(\tau_{\mathrm{N}}\right)_{n^{\prime}}\left(l^{\prime}, p^{\prime}\right)} q_{r}\left(n^{\prime}\right) w_{k^{\prime}} w_{n^{\prime}}+\frac{C_{n}(l, p) q_{r}(n)}{\omega_{n}(l, p)}\left(\mu_{k} T_{\mathrm{loc}, \mathrm{N}} u_{x}+\eta_{k} T_{\mathrm{loc}, \mathrm{N}} u_{y}\right),
\end{gathered}
$$




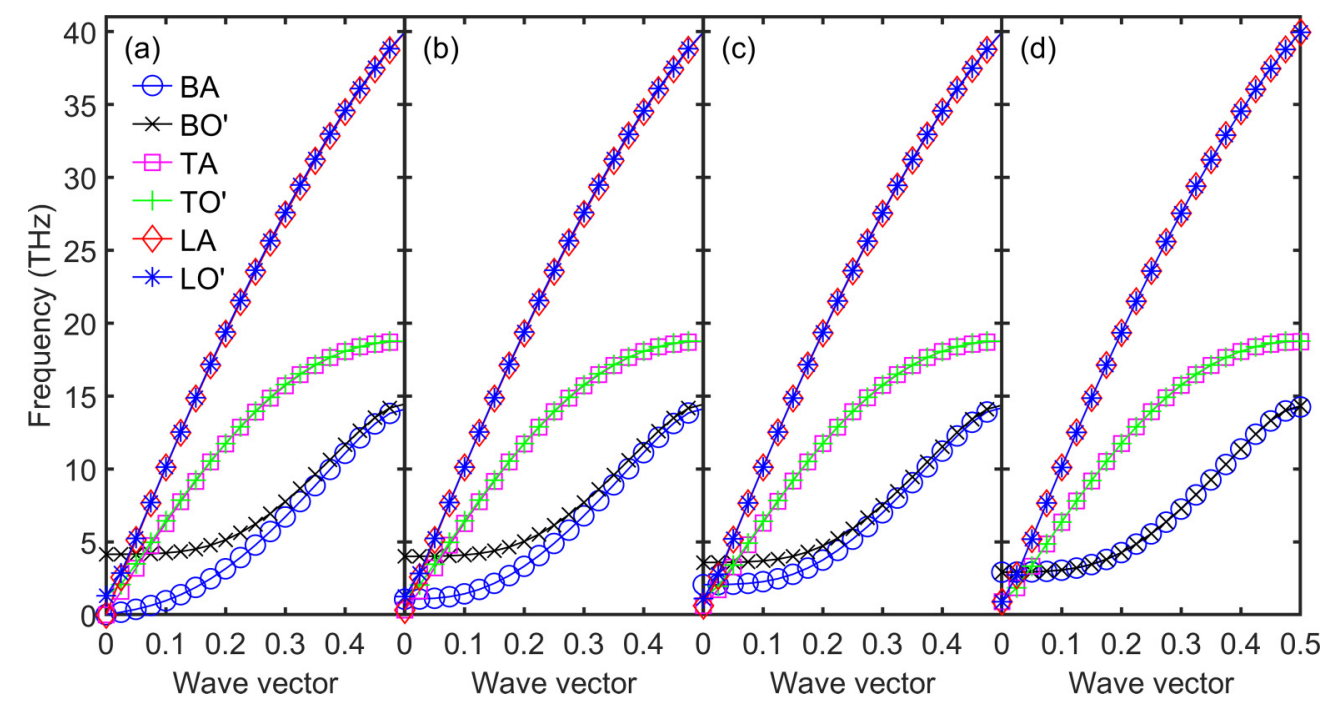

FIG. 15. Phonon dispersion of graphite at different dimensionless $q_{z}$ from $\Gamma$ to $A$ points in the first Brillouin zone: (a) $Q_{z}=0$, (b) $Q_{z}=\frac{1}{6}$, (c) $Q_{z}=\frac{1}{3}$, and (d) $Q_{z}=\frac{1}{2}$. Six phonon branches along the $\Gamma-M$ direction for $Q_{z}=0$ and along the directions parallel to $\Gamma-M$ within $\Gamma A L M$ for other $Q_{z}$ [c.f. Fig. 2(a)] are shown.

where $C_{n}(l, p)$ and $\omega_{n}(l, p)$ are the discrete forms of the modal heat capacity $C_{\mathbf{q} p}$ and frequency $\omega_{\mathbf{q} p}$, and the explicit expressions of the phonon drift velocity components are, respectively,

$$
\begin{aligned}
& T_{\mathrm{loc}, \mathrm{N}} u_{x}=\frac{1}{\left(\mathbf{C}_{\tau_{\mathrm{N}}}^{1}\right)_{x x}} F \sum_{p^{\prime}} \sum_{l^{\prime}} \sum_{k^{\prime}=1}^{N_{\theta}} \sum_{n^{\prime}=1}^{N_{q r}} \frac{\left[q_{r}\left(n^{\prime}\right)\right]^{2} \mu_{k^{\prime}}}{\omega_{n^{\prime}}\left(l^{\prime}, p^{\prime}\right)} \frac{\phi_{n^{\prime}}^{k^{\prime}}\left(l^{\prime}, p^{\prime}\right)}{\left(\tau_{\mathrm{N}}\right)_{n^{\prime}}\left(l^{\prime}, p^{\prime}\right)} w_{k^{\prime}} w_{n^{\prime}}, \\
& T_{\mathrm{loc}, \mathrm{N}} u_{y}=\frac{1}{\left(\mathbf{C}_{\tau_{\mathrm{N}}}^{1}\right)_{y y}} F \sum_{p^{\prime}} \sum_{l^{\prime}} \sum_{k^{\prime}=1}^{N_{\theta}} \sum_{n^{\prime}=1}^{N_{q r}} \frac{\left[q_{r}\left(n^{\prime}\right)\right]^{2} \eta_{k^{\prime}}}{\omega_{n^{\prime}}\left(l^{\prime}, p^{\prime}\right)} \frac{\phi_{n^{\prime}}^{k^{\prime}}\left(l^{\prime}, p^{\prime}\right)}{\left(\tau_{\mathrm{N}}\right)_{n^{\prime}}\left(l^{\prime}, p^{\prime}\right)} w_{k^{\prime}} w_{n^{\prime}} .
\end{aligned}
$$

The step scheme is adopted for the spatial discretization of the phonon Boltzmann Eq. (D1), with a discrete form in the first quadrant of the angular space $(\theta \in[0, \pi / 2]$, i.e., $\mu>0, \quad \eta>0)$ written as

$$
\mu_{k} \frac{\phi_{n, i, j}^{k}(l, p)-\phi_{n, i-1, j}^{k}(l, p)}{\Delta x}+\eta_{k} \frac{\phi_{n, i, j}^{k}(l, p)-\phi_{n, i, j-1}^{k}(l, p)}{\Delta y}=\frac{\left(\phi_{\mathrm{R}}^{\mathrm{eq}}\right)_{n, i, j}(l, p)-\phi_{n, i, j}^{k}(l, p)}{\left(\Lambda_{\mathrm{R}}\right)_{n}(l, p)}+\frac{\left(\phi_{\mathrm{N}}^{\mathrm{eq}}\right)_{n, i, j}^{k}(l, p)-\phi_{n, i, j}^{k}(l, p)}{\left(\Lambda_{\mathrm{N}}\right)_{n}(l, p)}
$$

where $i=1,2, \ldots, N_{x}$, and $j=1,2, \ldots, N_{y}$ represent the indexes of discrete $x$ and $y$ coordinates, with the spatial steps being $\Delta x$ and $\Delta y$, respectively. The evolution equation for the discrete deviational energy distribution function is thus obtained from Eq. (D6) as

$$
\phi_{n, i, j}^{k}=\frac{M_{n}^{k} \phi_{n, i-1, j}^{k}+N_{n}^{k} \phi_{n, i, j-1}^{k}+\frac{\left(\Lambda_{\mathrm{C}}\right)_{n}}{\left(\Lambda_{\mathrm{R}}\right)_{n}}\left(\phi_{\mathrm{R}}^{\mathrm{eq}}\right)_{n, i, j}+\frac{\left(\Lambda_{\mathrm{C}}\right)_{n}}{\left(\Lambda_{\mathrm{N}}\right)_{n}}\left(\phi_{\mathrm{N}}^{\mathrm{eq}}\right)_{n, i, j}^{k}}{M_{n}^{k}+N_{n}^{k}+1},
$$

where $M_{n}^{k}(l, p)=\frac{\mu_{k}\left(\Lambda_{\mathrm{C}}\right)_{n}(l, p)}{\Delta x}$, and $N_{n}^{k}(l, p)=\frac{\eta_{k}\left(\Lambda_{\mathrm{C}}\right)_{n}(l, p)}{\Delta y}$ are introduced for short notations, and the overall basal plane MFP is defined as $1 / \Lambda_{\mathrm{C}}=1 / \Lambda_{\mathrm{R}}+1 / \Lambda_{\mathrm{N}}$. The spatial discretization of the phonon Boltzmann equation and the evolution equations of discrete phonon distribution functions are similar in the other three quadrants, which could be found in Ref. [30].

Once the discrete deviational energy distribution is resolved, the macroscopic field variables are calculated by the discrete forms of Eqs. (11) and (12) as

$$
\begin{gathered}
C_{V}\left[T(i, j)-T_{0}\right]=F \sum_{p} \sum_{l} \sum_{k=1}^{N_{\theta}} \sum_{n=1}^{N_{q r}} \phi_{n, i, j}^{k}(l, p) q_{r}(n) w_{k} w_{n}, \\
J_{x}(i, j)=F \sum_{p} \sum_{l} \sum_{k=1}^{N_{\theta}} \sum_{n=1}^{N_{q r}}\left(v_{g r}\right)_{n}(l, p) \mu_{k} \phi_{n, i, j}^{k}(l, p) q_{r}(n) w_{k} w_{n}, \\
J_{y}(i, j)=F \sum_{p} \sum_{l} \sum_{k=1}^{N_{\theta}} \sum_{n=1}^{N_{q r}}\left(v_{g r}\right)_{n}(l, p) \eta_{k} \phi_{n, i, j}^{k}(l, p) q_{r}(n) w_{k} w_{n} .
\end{gathered}
$$



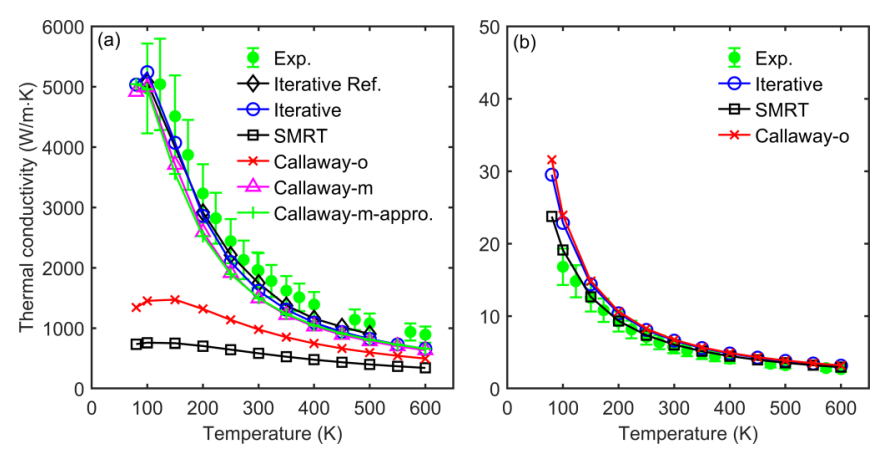

FIG. 16. Temperature-dependent bulk thermal conductivity of graphite with natural abundancy $\left(1.1 \%{ }^{13} \mathrm{C}\right)$ : (a) basal plane result and (b) $c$-axis result. The line with circles denotes the iterative solution of phonon Boltzmann equation with full scattering term; the line with squares denotes the single mode relaxation time (SMRT) approximation; the line with cross symbols (or triangles) denotes the Callaway's dual relaxation model with the original (-o) definition [or modified (-m) definition in Eq. (13)] of normal and Umklapp scattering rates, whereas the line with plus symbols denotes the Callaway's model with the modified definition of normal and Umklapp scattering rates under the approximations made in Sec. II B 3. The line with diamonds refers to the iterative solution from Ref. [35] with the same pseudopotential and van der Waals (vdW) functional. The discrete filled circles with error bars denote the experimental results from Ref. [60].

Throughout this paper, several types of boundaries are encountered: isothermal and adiabatic boundaries, and the periodic heat flux boundary. The isothermal boundary is treated by the following phonon black-body emission scheme:

$$
\begin{aligned}
& \phi\left(x=0, \mu>0, q_{r}, q_{z}, p\right)=C_{\mathbf{q} p}\left(T_{h}-T_{0}\right) \\
& \phi\left(x=L, \mu<0, q_{r}, q_{z}, p\right)=C_{\mathbf{q} p}\left(T_{c}-T_{0}\right) .
\end{aligned}
$$

The adiabatic boundary is treated by the fully diffuse scheme as follows:

$$
\begin{array}{r}
\phi\left(x, y=0, \eta>0, q_{r}, q_{z}, p\right) \\
=\frac{-\int_{\pi}^{2 \pi} \eta^{\prime} \phi\left(x, y=0, \eta^{\prime}, q_{r}, q_{z}, p\right) d \theta}{\int_{0}^{\pi} \eta^{\prime} d \theta}, \\
\phi\left(x, y=W, \eta<0, q_{r}, q_{z}, p\right) \\
=\frac{-\int_{0}^{\pi} \eta^{\prime} \phi\left(x, y=W, \eta^{\prime}, q_{r}, q_{z}, p\right) d \theta}{\int_{\pi}^{2 \pi} \eta^{\prime} d \theta},
\end{array}
$$

where $\eta^{\prime}=\sin \theta$. To implement a constant temperature gradient in the in-plane heat transport, the following periodic heat flux boundary is adopted:

$$
\begin{aligned}
& \phi\left(x=0, \mu>0, q_{r}, q_{z}, p\right) \\
& \quad=C_{\mathbf{q} p}\left(T_{h}-T_{c}\right)+\phi\left(x=L, \mu>0, q_{r}, q_{z}, p\right) \\
& \phi\left(x=L, \mu<0, q_{r}, q_{z}, p\right) \\
& \quad=C_{\mathbf{q} p}\left(T_{c}-T_{h}\right)+\phi\left(x=0, \mu<0, q_{r}, q_{z}, p\right) .
\end{aligned}
$$

The DOM numerical solution is implemented through an iteration process $[30,61]$. Within each iteration step, the discrete deviational energy distribution function for $\theta \in[0, \pi / 2]$ is firstly updated from the left-bottom boundary of the system based on Eq. (D7). The update is similar in the other three quadrants of angular space [30]. Then the discrete equilibrium distribution functions of resistive and normal scatterings are computed based on Eqs. (D2)-(D5), and the macroscopic field variables are computed based on Eqs. (D8)-(D10). Before the next iteration step, boundary treatment is required for the deviational energy distribution function based on Eqs. (D11)(D14) [30,31]. The iteration process is terminated until the relative differences of macroscopic field variables between two successive iteration steps are $<10^{-6}$.
[1] W. E. Bron, Nonequilibrium Phonon Dynamics (Plenum Press, New York, 1985).

[2] H. Beck, P. Meier, and A. Thellung, Phys. Status Solidi A 24, 11 (1974).

[3] S. Lee and X. Li, in Nanoscale Energy Transport: Emerging Phenomena, Methods and Applications, edited by B. Liao (IOP Publishing, Bristol, 2020), pp. 1.

[4] R. A. Guyer and J. A. Krumhansl, Phys. Rev. 148, 778 (1966).

[5] Y. Guo and M. Wang, Phys. Rep. 595, 1 (2015).

[6] F. X. Alvarez, D. Jou, and A. Sellitto, J. Appl. Phys. 105, 014317 (2009).

[7] Y. Guo and M. Wang, Phys. Rev. B 97, 035421 (2018).

[8] M. Simoncelli, N. Marzari, and A. Cepellotti, Phys. Rev. X 10, 011019 (2020).

[9] L. Sendra, A. Beardo, P. Torres, J. Bafaluy, F. X. Alvarez, and J. Camacho, Phys. Rev. B 103, L140301 (2021).

[10] A. Cepellotti, G. Fugallo, L. Paulatto, M. Lazzeri, F. Mauri, and N. Marzari, Nat. Commun. 6, 6400 (2015).

[11] S. Lee, D. Broido, K. Esfarjani, and G. Chen, Nat. Commun. 6, 6290 (2015).
[12] S. Huberman, R. A. Duncan, K. Chen, B. Song, V. Chiloyan, Z. Ding, A. A. Maznev, G. Chen, and K. A. Nelson, Science 364, 375 (2019).

[13] Z. Yan, G. Liu, J. Khan, and A. Balandin, Nat. Commun. 3, 827 (2012).

[14] H. Han, Y. Zhang, N. Wang, M. K. Samani, Y. Ni, Z. Y. Mijbil, M. Edwards, S. Xiong, K. Sääskilahti, M. Murugesan, Y. Fu, L. Ye, H. Sadeghi, S. Bailey, Y. A. Kosevich, C. J. Lambert, J. Liu, and S. Volz, Nat. Commun. 7, 11281 (2016).

[15] L. Shi, Science 364, 332 (2019).

[16] G. Fugallo, A. Cepellotti, L. Paulatto, M. Lazzeri, N. Marzari, and F. Mauri, Nano Lett. 14, 6109 (2014).

[17] L. Lindsay, W. Li, J. Carrete, N. Mingo, D. A. Broido, and T. L. Reinecke, Phys. Rev. B 89, 155426 (2014).

[18] C. D. Landon and N. G. Hadjiconstantinou, J. Appl. Phys. 116, 163502 (2014).

[19] X. Li and S. Lee, Phys. Rev. B 97, 094309 (2018).

[20] Y. Machida, N. Matsumoto, T. Isono, and K. Behnia, Science 367, 309 (2020).

[21] J. Callaway, Phys. Rev. 113, 1046 (1959).

[22] J. Ma, W. Li, and X. Luo, Phys. Rev. B 90, 035203 (2014). 
[23] M. J. Fryer and H. Struchtrup, Continuum Mech. Thermodyn. 26, 593 (2014).

[24] A. K. Majee and Z. Aksamija, Phys. Rev. B 93, 235423 (2016).

[25] S. Lee and L. Lindsay, Phys. Rev. B 95, 184304 (2017).

[26] Z. Ding, J. Zhou, B. Song, V. Chiloyan, M. Li, T.-H. Liu, and G. Chen, Nano Lett. 18, 638 (2018).

[27] A. K. Majee and Z. Aksamija, Phys. Rev. B 98, 024303 (2018).

[28] R. Yang, S. Yue, and B. Liao, Nanoscale Microscale Thermophys. Eng. 23, 25 (2019).

[29] M.-Y. Shang, C. Zhang, Z. Guo, and J.-T. Lü, Sci. Rep. 10, 8272 (2020).

[30] Y. Guo and M. Wang, Phys. Rev. B 96, 134312 (2017).

[31] X.-P. Luo, Y.-Y. Guo, M.-R. Wang, and H.-L. Yi, Phys. Rev. B 100, 155401 (2019).

[32] S. Lee, X. Li, and R. Guo, Nanoscale Microscale Thermophys. Eng. 23, 247 (2019).

[33] B.-D. Nie and B.-Y. Cao, Nanoscale Microscale Thermophys. Eng. 24, 94 (2020).

[34] E. Hazrati, G. A. de Wijs, and G. Brocks, Phys. Rev. B 90, 155448 (2014).

[35] Z. Ding, J. Zhou, B. Song, M. Li, T.-H. Liu, and G. Chen, Phys. Rev. B 98, 180302(R) (2018).

[36] X. Li and S. Lee, Phys. Rev. B 99, 085202 (2019).

[37] R. Gurzhi, Sov. Phys. JETP 19, 490 (1964).

[38] R. Gurzhi, Sov. Phys. Uspekhi 11, 255 (1968).

[39] J.-P. M. Péraud and N. G. Hadjiconstantinou, Phys. Rev. B 84, 205331 (2011).

[40] P. Giannozzi, S. Baroni, N. Bonini, M. Calandra, R. Car, C. Cavazzoni, D. Ceresoli, G. L. Chiarotti, M. Cococcioni, and I. Dabo, J. Phys.: Condens. Matter 21, 395502 (2009).

[41] N. Marzari, D. Vanderbilt, A. De Vita, and M. C. Payne, Phys. Rev. Lett. 82, 3296 (1999).

[42] Y. Baskin and L. Meyer, Phys. Rev. 100, 544 (1955).
[43] W. Li, J. Carrete, N. A. Katcho, and N. Mingo, Comput. Phys. Commun. 185, 1747 (2014).

[44] R. D'Souza and S. Mukherjee, Phys. Rev. B 96, 205422 (2017).

[45] V. Yu. Davydov, Yu. E. Kitaev, I. N. Goncharuk, A. N. Smirnov, J. Graul, O. Semchinova, D. Uffmann, M. B. Smirnov, A. P. Mirgorodsky, and R. A. Evarestov, Phys. Rev. B 58, 12899 (1998).

[46] L. Lindsay, D. A. Broido, and N. Mingo, Phys. Rev. B 83, 235428 (2011).

[47] S. Ghosh, W. Bao, D. L. Nika, S. Subrina, E. P. Pokatilov, C. N. Lau, and A. A. Balandin, Nat. Mater. 9, 555 (2010).

[48] R. Yang and G. Chen, Phys. Rev. B 69, 195316 (2004).

[49] A. J. McGaughey, E. S. Landry, D. P. Sellan, and C. H. Amon, Appl. Phys. Lett. 99, 131904 (2011).

[50] X.-P. Luo and H.-L. Yi, Int. J. Heat Mass Transfer 114, 970 (2017).

[51] M.-H. Bae, Z. Li, Z. Aksamija, P. N. Martin, F. Xiong, Z.-Y. Ong, I. Knezevic, and E. Pop, Nat. Commun. 4, 1734 (2013).

[52] R. A. Guyer and J. A. Krumhansl, Phys. Rev. 148, 766 (1966).

[53] Y. Guo and M. Wang, Int. J. Therm. Sci. 171, 107178 (2022).

[54] L. D. Landau and E. M. Lifshitz, Fluid Mechanics (Elsevier Pte Ltd., Singapore, 2004).

[55] A. Majumdar, J. Heat Transfer 115, 7 (1993).

[56] V. A. Titarev, J. Comput. Phys. 231, 109 (2012).

[57] F. Sharipov, Rarefied Gas Dynamics: Fundamentals for Research and Practice (Wiley-VCH, Weinheim, 2016).

[58] A. Ziabari, P. Torres, B. Vermeersch, Y. Xuan, X. Cartoixà, A. Torelló, J.-H. Bahk, Y. R. Koh, M. Parsa, P. D. Ye, F. X. Alvarez, and A. Shakouri, Nat. Commun. 9, 255 (2018).

[59] J. Jeong, X. Li, S. Lee, L. Shi, and Y. Wang, Phys. Rev. Lett. 127, 085901 (2021).

[60] C. Y. Ho, R. W. Powell, and P. E. Liley, J. Phys. Chem. Ref. Data 1, 279 (1972).

[61] Y. Guo, Z. Zhang, M. Nomura, S. Volz, and M. Wang, Int. J. Heat Mass Transfer 169, 120981 (2021). 